\title{
NASH AND DOMINANT STRATEGY IMPLEMENTATION IN ECONOMIC ENVIRONMENTS*
}

\author{
Jean-Jacques LAFFONT \\ Université de Toulouse, 31070 Toulouse, France \\ Ecole des Hautes Etudes en Sciences Sociales, 75006 Paris, France \\ Eric MASKIN \\ Massachusetts Institute of Technology, Cambridge, MA 02139, USA \\ University of Cambridge, Cambridge CD3 9DD, UK
}

\begin{abstract}
When environments are 'rich', single-valued social choice functions which are implementable in Nash strategies are implementable in dominant strategies. Moreover the Gibbard-Satterthwaite impossibility theorem of implementation in dominant strategies has been extended to differential economic environments. Therefore it is important to study implementation for non-rich environments. We characterize for quasi-linear utility functions mechanisms which are implementable in dominant strategies (providing a generalization of the Groves-Clarke mechanisms) and in Nash strategies. This second type of mechanisms differ from the first only by the types of transfers they allow. Properties of these mechanisms such as balancedness, individual rationality and robustness with respect to coalitions are then studied.
\end{abstract}

\section{Introduction}

A social choice rule is a correspondence that assigns to each profile of preferences that individuals might have a set of social alternatives or 'welfare optima'. A game (or mechanism) is said to implement a social choice rule if, for all profiles, the equilibrium outcomes of the game coincide with the welfare optima. Of course, the nature of the equilibrium outcomes depends on the solution concept adopted. In this paper, we shall concentrate on implementation in Nash and dominant strategy equilibrium.

The fundamental theorem on dominant strategy implementation is due to Gibbard (1973) and Satterthwaite (1975). It states that with unrestricted preferences, if the social choice rule is single-valued (i.e., there is a unique optimum for each profile of preferences) and has a range of at least three alternatives, then it is implementable in dominant strategies if and only if it is dictatorial. This negative result was extended to pure exchange economic environments by Dasgupta, Hammond and Maskin (1979) (later referred to as

*We are grateful to $P$. Champsaur and $\mathbf{H}$. Moulin for useful suggestions. 
DHM) and to more general, differential environments by Satterthwaite and Sonnenschein (1981).

Because Nash equilibrium is a considerably weaker solution concept than that of dominant strategies, results have been more encouraging for Nash implementation. In particular, Groves and Ledyard (1977) showed that, in economies with public goods, games can be constructed whose Nash equilibria are Pareto optimal. Hurwicz (1979a) refined this result by insisting that the Nash equilibria be Lindahl equilibria. Maskin (1977) derived the general theorem that any social choice rule satisfying monotonicity and veto-proof conditions (described bclow) is Nash implementable.

In contrast with these optimistic conclusions, Roberts (1979) demonstrated, in an important paper, that, with unrestricted preferences, any single-valued social choice correspondence that is Nash implementable is also implementable in dominant strategies. In such cases, nothing is gained by substituting the Nash equilibrium concept for dominant strategies. The result was extended by DHM to any domain of preferences sufficiently 'rich' (defined below).

Although the assumption of single-valuedness is very strong, it has considerable appeal. Less satisfactory for many applications, however, is the hypothesis of a rich domain. In particular, the family of 'quasi-linear' preferences (defined below), used extensively in the public incentives literature, is not rich. In this paper, we explore the possibilities of Nash versus dominant strategy implementation for this family of preferences. In section 2 , we define our terminology and review some of the existing literature. In the third section, we study Nash and dominant strategy implementation for an economy with convex, quasi-linear preferences over public and private goods. In this context, a social choice rule consists of a public decision function (a rule which assigns a level of public goods for each profile of preferences) and a vector of private transfer functions (rules which assign transfers of private goods to consumers for each profile). We find that although Nash and dominant stratcgics lead to the same class of implementable public decision functions, they imply rather different transfer functions. We characterize both Nash and dominant strategy implementable social choice rules. Finally, in sections 4,5 and 6 we study collusion by coalitions and the possibility of implementing balanced and individually rational social choice rules.

\section{Preliminaries}

We consider an economy defined by a finite set of agents $1, \ldots, n$ and a (nonempty) set $X$ of social alternatives. Each agent $i$ has a preference ordering $R_{i}$ on $X$. $R_{i}$ is assumed to belong to a family $\mathscr{R}_{i}$ of admissible preferences orderings. A social choice rule is a correspondence $F$ which associates with each profile of preferences $\boldsymbol{R}=\left(R_{1}, \ldots, R_{n}\right)\left(\in \prod_{i=1}^{n} \mathscr{R}_{i}\right)$ a (non-empty) subset $A \subseteq X$. One can interpret $A$ as the 'best' alternatives or welfare optima given the preferences $R . F$ 
is called single-valued if $\boldsymbol{F}(\boldsymbol{R})$ is a singleton for every profile $\boldsymbol{R}$. A mechanism is a product $\prod_{i=1}^{n} S_{i}$ of agents' strategy spaces and a function

$$
g: \prod_{i=1}^{n} S_{i} \rightarrow X
$$

which specifies an outcome for any $n$-tuple of strategies chosen by agents.

A dominant strategy equilibrium of a mechanism $g: \prod_{i=1}^{n} S_{i} \rightarrow X$ for a preference profile $\boldsymbol{R}$ is an $n$-tuple of strategies $\left(s_{1}^{*}, \ldots, s_{n}^{*}\right) \in \prod_{i} S_{i}$ which each agent $i$ is willing to use regardless of the strategies chosen by others. That is,

$$
\forall i, \quad \forall s_{i} \in S_{i}, \quad \forall s_{-i} \in \prod_{j \neq i} S_{j}, \quad g\left(s_{i}^{*}, s_{-i}\right) R_{i} g\left(s_{i}, s_{-i}\right),
$$

where

$$
s_{-i}=\left(s_{1}, \ldots, s_{i-1}, s_{i+1}, \ldots, s_{n}\right)
$$

and

$$
\left(s_{i}^{*}, s_{-i}\right)=\left(s_{1}, \ldots, s_{i-1}, s_{i}^{*}, s_{i+1}, \ldots, s_{n}\right)
$$

Suppose that for all $i, S_{i}=\mathscr{R}_{i}$. In this case, $g$ is called a direct revelation mechanism because a strategy consists of announcing a preference ordering. If, for all $\left(R_{1}, \ldots, R_{n}\right) \in \prod_{i=1}^{n} \mathscr{R}_{i},\left(s_{1}, \ldots, S_{n}\right)=\left(R_{1}, \ldots, R_{n}\right)$ constitutes a dominant strategy equilibrium and, furthermore, $g\left(s_{1}, \ldots, s_{n}\right) \in F\left(R_{1}, \ldots, R_{n}\right)$, then $g$ partially implements $F$ truthfully in dominant strategies. If, moreover, $\forall\left(R_{1}, \ldots, R_{n}\right) \in \prod_{i=1}^{n} \mathscr{R}_{i} \forall a \in f\left(R_{1}, \ldots, R_{n}\right)$ there exists $g: \prod_{i=1}^{n} \mathscr{R}_{i} \rightarrow X$ such that $g$ (partially) implements $F$ truthfully in dominant strategies and $g\left(R_{1}, \ldots, R_{n}\right)=a$, then $F$ is truthfully implementable in dominant strategies.

It is straightforward to characterize those single-valued social choice rules that can be implemented truthfully in dominant strategies. We shall say that a social choice rule $F$ satisfies independent person-by-person monotonicity (IPM) if

$$
\begin{aligned}
& \forall x, y \in X, \quad \forall\left(R_{1}, \ldots, R_{n}\right), \quad \forall i, \quad \forall R_{i}^{\prime} \text { such that } x P_{i}^{\prime} y \\
& {\left[x \in F\left(R_{1}, \ldots, R_{n}\right) \rightarrow y \notin F\left(R_{i}^{\prime}, R_{-i}\right)\right] .}
\end{aligned}
$$

We then have:

Theorem 1 [DHM(1979)]. F is truthfully implementable in dominant strategies if and only if $\forall\left(R_{1}, \ldots, R_{n}\right), \forall a \in A$, there exists a single-valued selection $F^{*}$ of $F$ satisfying $I P M$ and $F^{*}\left(R_{1}, \ldots, R_{n}\right)=a$.

One difficulty with the concept of truthful implementation is that it does not preclude individuals having non-truthful dominant strategies corresponding to 
outcomes outside the social choice set. We shall say that $F$ is strictly truthfully implementable in dominant strategies if the mechanisms $g$ in the definition of truthful implementation can be chosen to have no untruthful dominant strategies. $^{1}$

One way to ensure strictly truthful implementation is to hypothesize convex preferences and sufficient variability of the social choice rule. The following result is adapted from Laffont and Maskin (1981).

Theorem 2. Let $X$ be a subset of Euclidean space and for all $i$ let $\mathscr{R}_{i}$ be a domain of preference orderings over $X$. Suppose that, for each $i$ and $R_{i} \in \mathscr{R}_{i}, R_{i}$ is convex and differentiable. Assume too that the set $A\left(R_{i}, x\right)=\left\{y \in X \mid y R_{i} x\right\}$ has non-empty interior and that if $y \in \operatorname{int} A\left(R_{i}, x\right)$, then $y P_{i} x$ (i.e., indifference curves are not 'thick'). Suppose that $F$ is a single-valued choice rule such that $\forall\left(\bar{R}_{1}, \ldots, \bar{R}_{n}\right), \forall i$,

(i) $\forall R_{i}^{\prime} \neq \bar{R}_{i}, \quad \exists x \in\left\{F\left(\bar{R}_{i}, R_{-i}\right) \mid R_{-i} \in \prod_{j \neq i} \mathscr{R}_{j}\right\}$,

such that the tangents to $A\left(\bar{R}_{i}, x\right)$ and $A\left(R_{i}^{\prime}, x\right)$ at $x$ do not coincide.

(ii) $\left\{F\left(R_{i}, \bar{R}_{-i}\right) \mid R_{i} \in \mathscr{R}_{i}\right\}$ has a unique tangent hyperplane at each point.

Then, if $F$ is truthfully implementable in dominant strategies only truthful strategies are dominant, i.e., $F$ is strictly truthfully implementable.

Remark 1. The same result holds for non-single-valued social choice rules if we stipulate that (i) and (ii) hold for each single-valued selection that satisfies IPM.

Remark 2. Note that condition (i) is automatically satisfied if

$$
\forall i, \quad \forall \bar{R}_{i}, \quad\left\{F\left(\bar{R}_{i}, R_{-i}\right) \mid R_{-i} \in \prod_{j \neq i} \mathscr{R}_{j}\right\}=X .
$$

Proof. Suppose that $F$ satisfies the hypotheses of the theorem. Suppose that for some $i, \bar{R}_{i}$, and $R_{i}^{\prime} \neq \bar{R}_{i}, R_{i}^{\prime}$ is a dominant strategy for agent $i$ with preferences $\bar{R}_{i}$ in the game form that truthfully implements $F$. From (i) there exist $\bar{R}_{-i}$ and $x$ such that $x=F\left(R_{i}^{\prime}, \bar{R}_{-i}\right)$ and such that the tangents to $A\left(\bar{R}_{i}, x\right)$ and $A\left(R_{i}^{\prime}, x\right)$ at $x$ do not coincide. From (ii) there exists a unique hyperplane $L$ that is tangent to

$$
B=\left\{F\left(R_{i}, \bar{R}_{-i}\right) \mid R_{i} \in \mathscr{R}_{i}\right\}
$$

at $x$. If $L$ is not tangent to $A\left(R_{i}^{\prime}, x\right)$ at $x$, then because $A\left(R_{i}^{\prime}, x\right)$ is convex $\left(R_{i}^{\prime}\right.$ is convex) the interior of $A\left(R_{i}^{\prime}, x\right)$ intersects $B$. But this means that alternatives

\footnotetext{
${ }^{1}$ Actually, this is a bit stronger than necessary because it is not essential to rule out untruthful dominant strategies as long as they lead to the same outcome as the truthful one. However, the definition will do for our purposes.
} 
preferred to $x$ (under $R_{i}^{\prime}$ ) are attainable by varying $R_{i}$, contradicting $R_{i}^{\prime \prime} \mathrm{s}$ dominance. Therefore $L$ is tangent to $A\left(R_{i}^{\prime}, x\right)$ at $x$. Similarly, $L$ must be tangent to $A\left(\bar{R}_{i}, x\right)$ at $x$, a contradiction of the hypothesized non-coincidence of tangents. Thus, $R_{i}^{\prime}$ cannot be a dominant strategy after all. Q.E.D.

A Nash equilibrium for profile $\left(R_{1}, \ldots, R_{n}\right)$ of a mechanism $g$ is an $n$-tuple of strategies $\left(s_{1}^{*}, \ldots, s_{n}^{*}\right) \in \prod_{i} S_{i}$ such that

$$
\forall i, \quad \forall s_{i} \in S_{i}, \quad g\left(s_{i}^{*}, s_{-i}^{*}\right) R_{i} g\left(s_{i}, s_{-i}^{*}\right) .
$$

Let $N E_{g}(\boldsymbol{R})$ be the Nash equilibria for profile $\boldsymbol{R}$ of mechanism $g$. Then $g$ (partially) implements $F$ in Nash strategies if, for all $\boldsymbol{R}, g\left(N E_{g}(\boldsymbol{R})\right) \subset F(\boldsymbol{R})$ and $N E_{g}(\boldsymbol{R})$ is non-empty. $F$ is implementable in Nash strategies if $\forall \boldsymbol{R}, \forall a \in F(\boldsymbol{R})$, there exists a $g$ that (partially) implements $F$ such that $a \in N E_{g}(R)$.

To state the general theorem on Nash implementability, we need to define two additional properties of social choice rules. A social choice rule $F$ is monotonic if $\forall \boldsymbol{R}, \boldsymbol{R}^{\prime} \in \mathscr{R}$, if $x \in F(\boldsymbol{R})$ and if $\forall i, \forall y \in X,\left[x R_{i} y \rightarrow x R_{i}^{\prime} y\right]$ then $x \in F\left(\boldsymbol{R}^{\prime}\right) . F$ satisfies $n o$ veto power if $\left[\forall x \in X, \forall i\right.$, if $\exists \boldsymbol{R}$ such that $\forall j \neq i, \forall y \in X, x R_{j} y$, then $\left.x \in F(\boldsymbol{R})\right]$.

Monotonicity says that if an alternative $x$ is welfare optimal for some profile of preferences and if those preferences are then altered so that $x$ does not fall in relation to any other alternative in anyone's preference ordering, then $x$ remains welfare optimal. No veto power requires that any alternative that is top-ranked (i.e., weakly preferred to all other alternatives) by all individuals but one be welfare optimal.

Monotonicity is a stringent condition. No veto power, however, is automatically satisfied in economic environments with non-satiation and private goods, since no two agents will agree that any given alternative (i.e., allocation) is top-ranked (each would like all of any private good to himself).

The theorem characterizing Nash implementability is:

Theorem 3 [Maskin (1977)]. If $F$ is implementable in Nash strategies, it is monotonic. If $n \geqq 3$ and $F$ satisfies no veto power, the converse holds, and, moreover, there exists a mechanism $\mathrm{g}$, such that

$$
N E_{g}(\boldsymbol{R})=F(\boldsymbol{R}) \text { for all } \boldsymbol{R} \text {. }
$$

In general, Nash implementability does not imply dominant strategy implementability, nor does the converse hold. Nonetheless, in one important special case - when preference domains are sufficiently rich and social choice rules are single-valued - the former implication does hold. To express richness formally, suppose that $R$ and $R^{\prime}$ belong to the domain $\mathscr{R}_{i}$. Let $D_{a, b}\left(R, R^{\prime}\right)$ be the set of orderings $R^{\prime \prime}$ that are $a-b$ monotone with respect to $\left(R, R^{\prime}\right)$; i.e., such that $\forall c \in X, a R c \rightarrow a R^{\prime \prime} c$ and $b R^{\prime} c \rightarrow b R^{\prime \prime} c . \mathscr{R}=\prod_{i=1}^{n} \mathscr{R}_{i}$ is rich or monotonically closed if 
$\forall i, \forall R, R^{\prime} \in \mathscr{R}_{i}, \forall a, b \in X$, such that $a R b \rightarrow a R^{\prime} b$ and $a P b \rightarrow a P^{\prime} b, \mathscr{R}_{i} \cap D_{a, b}\left(R, R^{\prime}\right)$ $\neq \varnothing$. It should be clear that, for example, the unrestricted domain is rich and that trivially, so is any domain consisting of a single preference profile. It can also be shown [see DHM (1979)] that the domain consisting of all convex, monotonic, and continuous preferences for private good is rich as well.

We can now state:

Theorem 4 [DHM (1979)]. If the domain of preferences $\prod_{i=1}^{n} \mathscr{R}_{i}$ is rich and the social choice rule $F$ is single-valued and implementable in Nash strategies, it is truthfully implementable in dominant strategies.

Theorem 4 leads us inevitably to the conclusion that if Nash strategies are going to get us any further than do dominant strategies, we had better turn to domains that are not rich - at least, if we maintain the hypothesis of singlevaluedness. It is worthwhile considering two important classes of non-rich domains.

Example 1. Cobb-Douglas preferences for two commodities.

In fig. 1, two sets of indifference curves are drawn. The $R_{\alpha}$ indifference curves correspond to the utility function $X_{1}^{\alpha} X_{2}^{1-\alpha}$, whereas the $R_{\beta}$ indifference curves

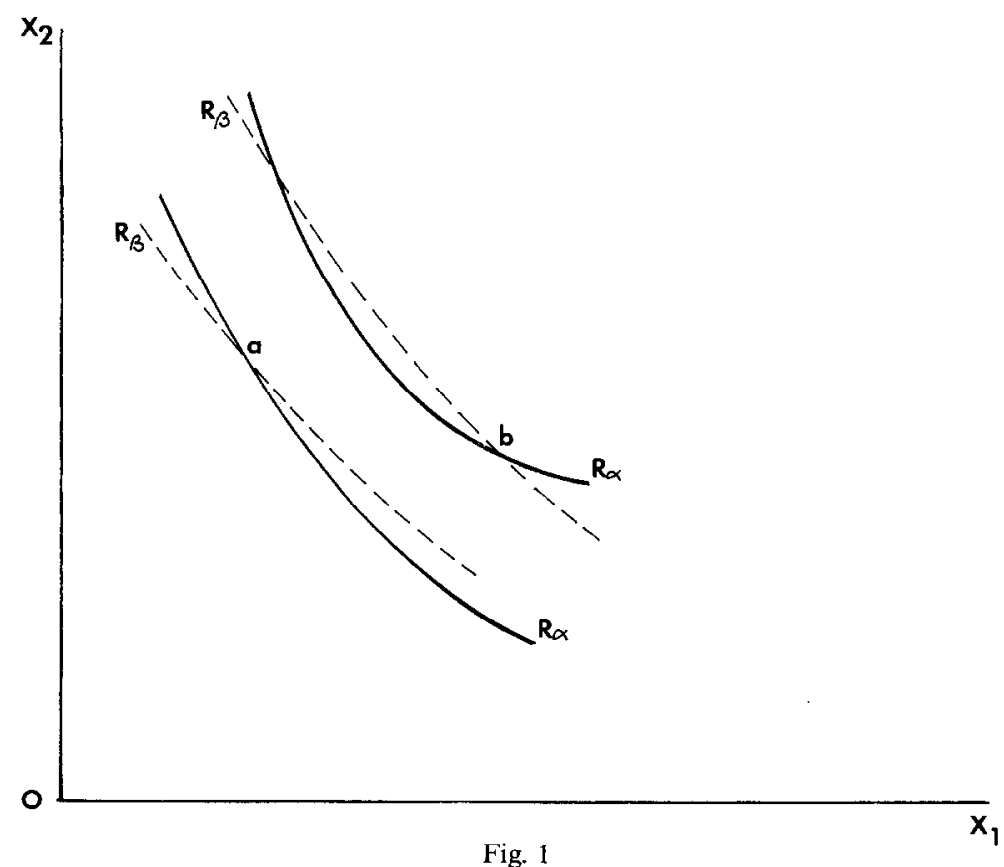


represent $X_{1}^{\beta} X_{2}^{1-\beta}$. If the domain of Cobb-Douglas preferences is monotonically closed, it must contain ordering $R^{\prime \prime}$ such that, for all $c, a R_{\alpha} c \rightarrow a R^{\prime \prime} c$ and $b R_{\beta} c \rightarrow b R^{\prime \prime} c$. But the former implication implies that $R_{\alpha}=R^{\prime \prime}$ and the latter, $R_{\beta}=R^{\prime \prime}$, a contradiction. Thus, the Cobb-Douglas domain is not rich.

From Hurwicz (1972) we known that there is no dominant strategy mechanism that truthfully implements the competitive equilibrium correspondence of a Cobb-Douglas exchange economy. On the other hand, this correspondence is monotonic and, vacuously, satisfies no veto power, so is implementable in Nash strategies (Theorem 3). Thus the non-richness of the domain does allow us to avoid the negativism of Theorem 3 .

Example 2. Quasi-linear preferences for two commodities.

Consider utility functions of the form

$$
v\left(X_{1}\right)+X_{2} .
$$

(Assume that $v$ is concave and has a local maximum.)

Notice that the indifference curves for such a function can be derived from one another by vertical translations. Consider the sets of indifference curves, corresponding to two different $v$ 's. In fig. 2 , the solid indifference curves correspond to utility function $v_{1}\left(X_{1}\right)+X_{2}$ (preference ordering $R_{1}$ ) whereas the dotted curves correspond to $v_{2}\left(X_{1}\right)+X_{2}$ (preference ordering $R_{2}$ ).

If the class of quasi-linear preferences is rich (monotonically closed), then there must exist a third quasi-linear preference ordering $R_{3}$ such that

$$
\begin{aligned}
& \forall c, \quad a R_{1} c \rightarrow a R_{3} c, \\
& \forall c, \quad b R_{2} c \rightarrow b R_{3} c .
\end{aligned}
$$

Because the $R_{3}$ indifference curve through $b$ must lie on or above the $R_{2}$ indifference curve through $b$, the vertical distance between the $R_{3}$ indifference curves through $a$ and $b$ at point $a$ must be less than or equal to $d_{2}$, the vertical distance between the $R_{2}$ indifference curves. But because the $R_{3}$ indifference curve through $a$ must lie on or above the $R_{1}$ indifference curve through $a$, the vertical distance between the $R_{3}$ indifference curves at point $b$ must be at least $d_{1}$, the vertical distance between the $R_{1}$ indifference curves. Since $d_{1}>d_{2}$, the vertical distance between the $R_{3}$ indifference curves cannot remain constant, contradicting quasi-linearity. Therefore, the class of quasi-linear preferences cannot be rich after all, and Theorem 3 is again not applicable. Indeed, we shall now see that with quasi-linear preferences the Nash and dominant strategy implementable social choice rules differ. 


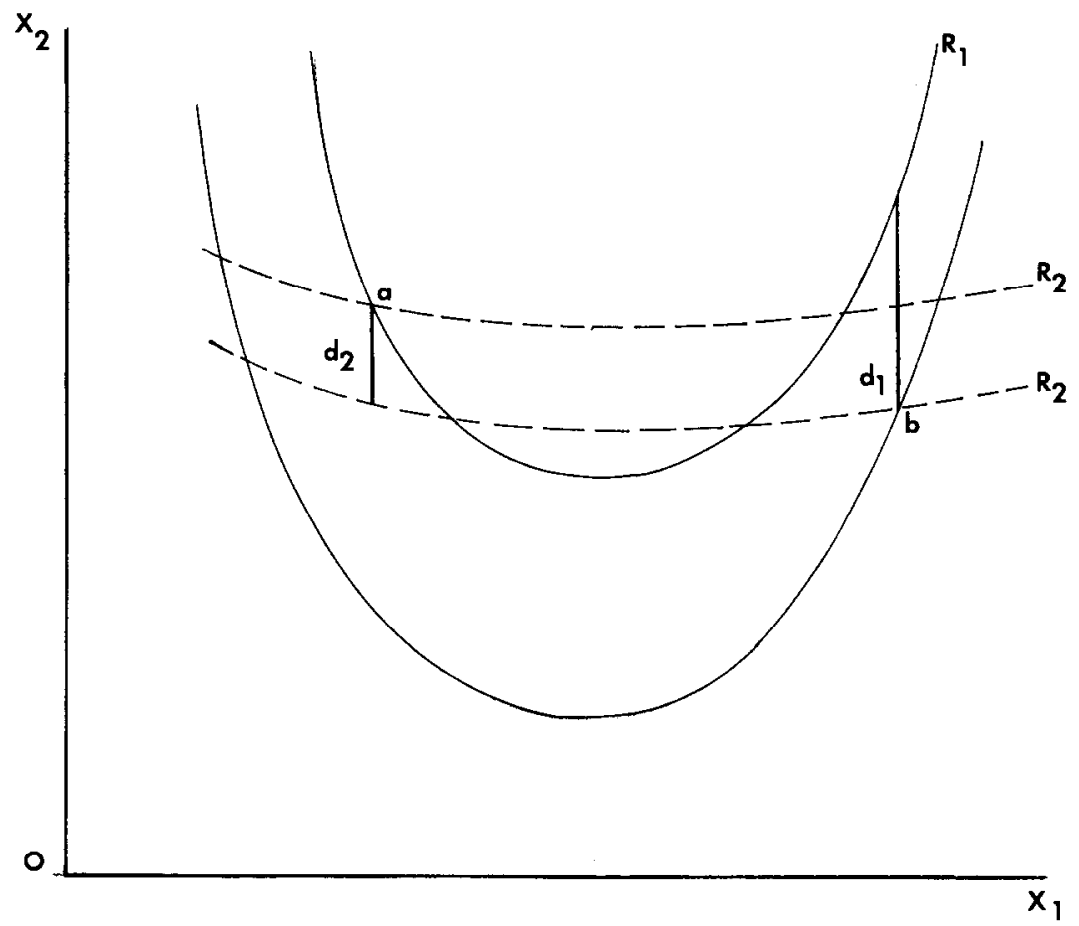

Fig. 2

\section{Implementation and quasi-linear preferences}

Consider an economy of $n$ agents and two goods, one public ${ }^{2}$ and one private. Suppose that the public good level can vary between 0 and 1 . Let $V$ be the class of all strictly concave differentiable functions on the interval $[0,1]$ taking their maximum in $(0,1)$. An agent with public good valuation $v \in V$ has preferences given by the utility function $v(K)+y$, where $K$ is the public good level and $y$ his consumption of private good. Let $L$ be the class of all linear functions. We are interested in studying the implementation of single-valued social choice rules when preferences are drawn from $V$. However, to facilitate our analysis, we shall work with the class $V^{*}=V \cup L$.

Consider a social choice rule $F$ from $\left(V^{*}\right)^{n}$ into $[0,1] \times \boldsymbol{R}^{n}$. For $\left(v_{1}, \ldots, v_{n}\right) \in\left(V^{*}\right)^{n}$, an element of $F\left(v_{1}, \ldots, v_{n}\right)$ is a vector $\left(K, t_{1}, \ldots, t_{n}\right)$, where $K$ denotes the public good level and $t_{i}$ the transfer to agent $i$. We shall asume that $F$ is single-valued when restricted to $V^{n}$.

${ }^{2}$ The restriction to a single public good is not essential (see the generalization after the proofs of Theorems 5 and 6). 
Let $F_{0}\left(v_{1}, \ldots, v_{n}\right)=\left\{K \mid \exists\left(t_{1}, \ldots, t_{n}\right)\right.$ with $\left.\left(K, t_{1}, \ldots, t_{n}\right) \in F\left(v_{1}, \ldots, v_{n}\right)\right\}$. We shall also assume that $F_{0}$ is upper hemicontinuous (where the metric on $V^{*}$ is the sup norm on $[0,1])$, weakly efficient [if $K \in F_{0}\left(v_{1}, \ldots, v_{n}\right)$ and $v_{1}=\cdots=v_{n}$, then $v_{i}(K) \geqq v_{i}(\widetilde{K})$ for all $i$ and $\left.\widetilde{K}\right]$, neutral $\left[\right.$ if $K \in F_{0}\left(v_{1}, \ldots, v_{n}\right)$, where $K \in(0,1)$ and $(K+c) \in(0,1)$ for constant $c$, then $(K+c) \in F_{0}\left(\tilde{v}_{1}, \ldots, \tilde{v}_{n}\right)$, where, for all $i, \tilde{v}_{i}(K)$ $\left.=v_{i}(K-c)\right]$, and convex-valued.

The principal results of this paper are the following:

Theorem 5. If (a) $F_{0}$ is upper hemicontinuous, weakly efficient, convex-valued and neutral, $(b)$ if $F$ is single-valued when restricted to $V^{n}$, and $(c)$ if $F$ is implementable in Nash strategies, then:

(i) there exists a continuous and semi-strictly increasing ${ }^{3}$ function $h$ from $\boldsymbol{R}^{n}$ into $\boldsymbol{R}$ such that $h(0, \ldots, 0)=0$, and

(ii) there exist functions $t_{i}^{*}$ from $[0,1] \times \boldsymbol{R}^{n}$ into $\boldsymbol{R}, i=1, \ldots, n$, such that for all $\left(v_{1}, \ldots, v_{n}\right) \in V^{n}$,

$$
\begin{aligned}
F\left(v_{1}, \ldots, v_{n}\right)= & \left\{\left(K, t_{1}, \ldots, t_{n}\right) \mid h\left(v_{1}^{\prime}(K), \ldots, v_{n}^{\prime}(K)\right)=0\right. \\
& \text { and } \left.t_{i}=t_{i}^{*}\left(K, v_{1}^{\prime}(K), \ldots, v_{n}^{\prime}(K)\right), \quad i=1, \ldots, n\right\} .
\end{aligned}
$$

Furthermore, the converse holds. ${ }^{4}$

Theorem 6. Suppose that $F$ satisfies (a) and $(b)$ of Theorem 5 and that $F$ is truthfully implementable in dominant strategies, then:

(iii) there exists a function $h$ as in Theorem 5, and

(iv) there exist functions $H_{i}$ from $V^{n-1}$ into $R, i=1, \ldots, n$, such that for all $\left(v_{1}, \ldots, v_{n}\right) \in V^{n}$

$$
\begin{aligned}
F\left(v_{1}, \ldots, v_{n}\right)= & \left\{\left(K, t_{1}, \ldots, t_{n}\right) \mid h\left(v_{1}^{\prime}(K), \ldots, v_{n}^{\prime}(K)\right)=0,\right. \\
& \text { for any } \left.i=1, \ldots, n, t_{i}=-\int_{0}^{K} h_{i}\left(v_{-i}^{\prime}(t)\right) \mathrm{d} t+H_{i}\left(v_{-i}\right)\right\}^{5},
\end{aligned}
$$

where $h_{i}: \boldsymbol{R}^{n-1} \rightarrow \boldsymbol{R}$ satisfies

$(* * *)$

$$
\begin{aligned}
& h\left(h_{i}\left(a_{-i}\right), a_{-i}\right)=0 \text { if } \exists a_{i} \text { such that } h\left(a_{i}, a_{-i}\right)=0, \\
& h_{i}\left(a_{-i}\right)=0, \text { otherwise. }
\end{aligned}
$$

Furthermore, the converse holds, ${ }^{4}$ and if in addition $h$ is strictly increasing, $F$ is strictly truthfully implementable in dominant strategies.

\footnotetext{
${ }^{3} \mathrm{By}$ 'semi-strictly increasing', we mean that if $x \gg \tilde{x}$, then $h(x)>h(\tilde{x})$.

${ }^{4}$ The converse is established only for the domain $V^{n}$.

${ }^{5} v_{i}=\left(v_{1}, \ldots, v_{i-1}, v_{i+1}, \ldots, v_{n}\right)$.
} 
Note that the class of implementable public decision correspondences (that is, the class of first components of implementable $F$ 's) is the same regardless of whether the solution concept is Nash or dominance. Furthermore, the public decisions are defined by functions of the derivatives of public good valuations.

If, in particular, $h=\sum_{i=1}^{n} \lambda_{i} v_{i}^{\prime}$, then the public decision $K$ maximizes $\sum_{i=1}^{n} \lambda_{i} v_{i}$. However, because $h$ depends only on the derivatives of the $v_{i}$ 's, a public decision which maximizes, say, $\sum \lambda_{i} \log v_{i}$, is not implementable.

Nash and dominant strategies differ in so far as the kinds of transfers they can implement. Nash implementation requires the transfers to be functions of the public decision and the derivatives of the public valuations. Dominant strategy implementation, on the other hand, makes an agent's transfer the sum of the two terms: a term depending on the derivatives of the public valuations and the public decision, and a term depending on the public valuation functions of other agents. For a given public decision correspondence, neither class of transfers is contained in the other, although the intersection of the two is non-empty.

Under dominant strategy implementation, the transfers obtained in Theorem 5 correspond to the so-called Clarke-Groves mechanisms [see Green and Laffont (1979)] when $h$ is linear,

$$
\begin{aligned}
t_{i} & =\int_{0}^{K} \sum_{j \neq i} \lambda_{j} v_{j}^{\prime}(t) \mathrm{d} t+H_{i}\left(v_{-i}(\cdot)\right) \\
& =\sum_{j \neq i} \lambda_{j} v_{j}(K)+H_{i}\left(v_{-i}(\cdot)\right) .
\end{aligned}
$$

Another example in the case of two agents is

$$
h\left(v_{1}^{\prime}, v_{2}^{\prime}\right)=\left(v_{1}^{\prime}\right)^{3}+v_{2}^{\prime}
$$

If then

$$
v_{1}(K)=-\theta_{1}\left(K-\theta_{2}\right)^{2}, \quad v_{2}(K)=\theta_{3} K,
$$

we obtain

$$
\theta_{3}=-\left(-2 \theta_{1}\left(K-\theta_{2}\right)\right)^{3},
$$

or

$$
K=\theta_{2}+\left(\theta_{3} / 2 \theta_{1}\right)^{1 / 3} .
$$

The proofs of Theorems 5 and 6 will be aided by a trio of lemmas:

Lemma 1. Suppose that $F_{0}$ satisfies $(a)$ and $(b)$ of Theorem 5. Then, for all $\left(v_{1}, \ldots, v_{n}\right),\left(\tilde{v}_{1}, \ldots, \tilde{v}_{n}\right) \in\left(V^{*}\right)^{n}$ such that $v_{i}^{\prime}=\tilde{v}_{i}^{\prime}$ (primes denote derivatives) for all $i$,

$$
F\left(v_{1}, \ldots, v_{n}\right)=F\left(\tilde{v}_{1}, \ldots, \tilde{v}_{n}\right),
$$


if $F$ is implementable in Nash strategies, and

$$
F_{0}\left(v_{1}, \ldots, v_{n}\right)=F_{0}\left(\tilde{v}_{1}, \ldots, \tilde{v}_{n}\right)
$$

if $F$ is truthfully implementable in dominant strategies.

Proof. First, suppose that $F$ is implementable in Nash strategies. Then, from Theorem $3, F$ is monotonic. Suppose that for $\left(v_{1}, \ldots, v_{n}\right),\left(\tilde{v}_{1}, \ldots, \tilde{v}_{n}\right) \in\left(V^{*}\right)^{n}, v_{i}^{\prime}=\tilde{v}_{i}^{\prime}$ for all $i$. If $a \in F\left(v_{1}, \ldots, v_{n}\right)$, then, from monotonicity, $a \in F\left(\tilde{v}_{1}, \ldots, \tilde{v}_{n}\right)$. (Since $v_{i}$ and $\tilde{v}_{i}$ differ only by a constant.)

Next, suppose that $F$ is truthfully implementable in dominant strategies. Consider $\left(v_{1}, \ldots, v_{n}\right)$ and $\left(\tilde{v}_{1}, \ldots, \tilde{v}_{n}\right)$ as hypothesized and suppose that $\tilde{K} \in F_{0}\left(\tilde{v}_{1}, \ldots, \tilde{v}_{n}\right)$, Consider a sequence $\left\{v_{1}^{s}\right\}$ such that $v_{1}^{s} \rightarrow v_{1}$ and $v_{1}^{s}$ is 'more concave' than $v_{1}$ (and $\tilde{v}_{1}$ ) at $\tilde{K}$. That is,

$$
\begin{aligned}
\forall K^{*}, t_{1}^{*}, & v_{1}(\tilde{K})+\tilde{t}_{1} \geqq v_{1}\left(K^{*}\right)+t_{1}^{*} \\
& \rightarrow v_{1}^{s}(\tilde{K})+\tilde{t}_{1} \geqq v_{1}^{s}\left(K^{*}\right)+t_{1}^{*},
\end{aligned}
$$

where the second inequality is strict if $\widetilde{K} \neq K^{*}$.

Let $\tilde{F}$ be a single-valued selection of $F$ satisfying IPM such that $\left(\tilde{K}, \tilde{t}_{1}, \ldots, \tilde{t}_{n}\right)$ $=\tilde{F}\left(\tilde{v}_{1}, \ldots, \tilde{v}_{n}\right)$. (By Theorem 1 such a selection exists.) For each $s$, let $\left(K^{s}, t_{1}^{s}, \ldots, t_{n}^{s}\right)$ $=\tilde{F}\left(v_{1}^{s}, \tilde{v}_{2}, \ldots, \tilde{v}_{n}\right)$. From IPM, $v_{1}^{s}\left(K^{s}\right)+t_{1}^{s} \geqq v_{1}^{s}(\widetilde{K})+\tilde{t}_{1}$. Thus, if $K^{s} \neq \tilde{K},(\alpha)$ implies that

$$
v_{1}\left(K^{s}\right)+t_{1}^{s}>v_{1}(\tilde{K})+\tilde{t}_{1}
$$

and

$$
\tilde{v}_{1}\left(K^{s}\right)+t_{1}^{s}>\tilde{v}_{1}(\tilde{K})+\tilde{t}_{1}
$$

But this last inequality violates IPM. Therefore, $K^{s}=\tilde{K}$ for all $s$. By continuity,

$$
\tilde{K} \in F_{0}\left(v_{1}, \tilde{v}_{2}, \ldots, \tilde{v}_{n}\right)
$$

Continuing iteratively, we conclude that $\tilde{K} \in F_{0}\left(v_{1}, \ldots, v_{n}\right)$. Hence, $F_{0}\left(\tilde{v}_{1}, \ldots, \tilde{v}_{n}\right) \subseteq F_{0}\left(v_{1}, \ldots, v_{n}\right)$. Similarly $F_{0}\left(v_{1}, \ldots, v_{n}\right) \subseteq F_{0}\left(\tilde{v}_{1}, \ldots, \tilde{v}_{n}\right)$ and thus $F_{0}\left(v_{1}, \ldots, v_{n}\right)=F_{0}\left(\tilde{v}_{1}, \ldots, \tilde{v}_{n}\right)$ Q.E.D.

Lemma 2. Suppose that $F$ satisfies $(a)$ and $(b)$ of Theorem 5 and is either implementable in Nash strategies or truthfully implementable in dominant strategies. Then, for $\left(w_{1}, \ldots, w_{n}\right) \in L^{n}$, if $F_{0}\left(w_{1}, \ldots, w_{n}\right)$ intersects $(0,1), F_{0}\left(w_{1}, \ldots, w_{n}\right)$ $=[0,1]$ and there exists no $\left(\tilde{w}_{1}, \ldots, \tilde{w}_{n}\right) \in L^{n}$ with $\tilde{w}_{i}^{\prime}>w_{i}^{\prime}$ for all $i$, such that $F_{0}\left(\tilde{w}_{1}, \ldots, \tilde{w}_{n}\right)$ intersects $(0,1)$. 
Proof. If for $K \in(0,1)$ and $\left(w_{1}, \ldots, w_{n}\right) \in L^{n}, K \in F_{0}\left(w_{1}, \ldots, w_{n}\right)$, then by $F$ 's neutrality, $\tilde{K} \in F_{0}\left(w_{1}, \ldots, w_{n}\right)$ for all $\tilde{K} \in(0,1)$ [if $K \in F_{0}\left(w_{1}, \ldots, w_{n}\right)$, then from neutrality $K+c \in F_{0}\left(\bar{w}_{1}, \ldots, \bar{w}_{n}\right)$ where $\bar{w}_{i}$ is $w_{i}$ shifted to the right by $c$. Since $\bar{w}_{i}^{\prime}$ $=w_{i}^{\prime}$, we conclude from Lemma 1 that $\left.K+c \in F_{0}\left(w_{1}, \ldots, w_{n}\right)\right]$. In particular, $\frac{1}{2} \in F_{0}\left(w_{1}, \ldots, w_{n}\right)$. Let $\left(\frac{1}{2}, t_{1}, \ldots, t_{n}\right) \in F\left(w_{1}, \ldots, w_{n}\right)$. Suppose that, for $\left(\tilde{w}_{1}, \ldots, \tilde{w}_{n}\right) \in L^{n}$ with $\tilde{w}_{i}^{\prime}>w_{i}^{\prime}, F_{0}\left(\tilde{w}_{1}, \ldots, \tilde{w}_{n}\right)$ intersects $(0,1)$. Then $\frac{1}{4} \in F_{0}\left(\tilde{w}_{1}, \ldots, \tilde{w}_{n}\right)$. For all $i$, choose $v_{i}^{\prime} \in V$ such that $v_{i}^{\prime}\left(\frac{1}{2}\right)=w_{i}^{\prime}$ and $v_{i}^{\prime}\left(\frac{1}{4}\right)=\tilde{w}_{i}^{\prime}$. Now, $v_{i}$ is 'more concave' than $w_{i}$ at $\frac{1}{2}$ in the sense that

$$
\begin{array}{r}
\forall K \in[0,1], \quad \forall \bar{t}_{i}, \overline{\bar{t}}_{i}, \quad w_{i}\left(\frac{1}{2}\right)+\bar{t}_{i} \geqq w_{i}(K)+\bar{t}_{i} \\
\Rightarrow v_{i}\left(\frac{1}{2}\right)+\bar{t}_{i} \geqq v_{i}(K)+\bar{t}_{i},
\end{array}
$$

where the latter inequality is strict if $K \neq \frac{1}{2}$.

Similarly, $v_{i}$ is more concave than $\tilde{w}_{i}$ at $\frac{1}{4}$,

$$
\begin{array}{r}
\forall K \in[0,1], \quad \forall \bar{t}_{i}, t_{i}, \quad \tilde{w}_{i}\left(\frac{1}{4}\right)+\bar{t}_{i} \geqq \tilde{w}_{i}(K)+t_{i} \\
\quad \Rightarrow v_{i}\left(\frac{1}{4}\right)+\bar{t}_{i} \geqq v_{i}(K)+t_{i},
\end{array}
$$

where, again, the second inequality is strict if $K \neq \frac{1}{4}$.

If $F$ is Nash implementable, then, from Theorem $3, F$ is monotonic. Hence, from (1) and (2) we conclude that since $\frac{1}{2} \in F_{0}\left(w_{1}, \ldots, w_{n}\right), \frac{1}{2} \in F_{0}\left(v_{1}, \ldots, v_{n}\right)$ and since $\frac{1}{4} \in F_{0}\left(\tilde{w}_{1}, \ldots, \tilde{w}_{n}\right), \frac{1}{4} \in F_{0}\left(v_{1}, \ldots, v_{n}\right)$. But $F$ was assumed to be single-valued if its arguments lie in $V$, a contradiction. Therefore, the lemma is established for a Nash implementable $F$.

Suppose, then, that $F$ is truthfully implementable in dominant strategies. Let $\tilde{F}$ be a single-valued selection of $F$ satisfying IPM and such that $\frac{1}{2}=\tilde{F}_{0}\left(w_{1}, \ldots, w_{n}\right)$. By an argument analogous to that of Lemma $1, \frac{1}{2}=\tilde{F}\left(v_{1}, \ldots, v_{n}\right)$. Hence $\frac{1}{2} \in F_{0}\left(v_{1}, \ldots, v_{n}\right)$. Similarly, $\frac{1}{4} \in F_{0}\left(v_{1}, \ldots, v_{n}\right)$. But $F\left(v_{1}, \ldots, v_{n}\right)$ is single-valued, since $\left(v_{1}, \ldots, v_{n}\right) \in V^{n}$, a contradiction. Q.E.D.

Suppose that $F$ satisfies the hypotheses of Lemma 2. Define $h: \boldsymbol{R}^{n} \rightarrow \boldsymbol{R}$ such that

$$
\left\{\begin{array}{l}
h\left(c_{1}, \ldots, c_{n}\right)=c, \text { such that } \exists\left(w_{1}, \ldots, w_{n}\right) \in L^{n}, \quad \text { with } \\
\frac{1}{2} \in F_{0}\left(w_{1}, \ldots, w_{n}\right) \text { and }\left(c_{1}, \ldots, c_{n}\right)=\left(w_{1}^{\prime}+c, \ldots, w_{n}^{\prime}+c\right) .
\end{array}\right.
$$

For $c$ sufficiently small, $\left(c_{1}-c, \ldots, c_{n}-c\right)>(0, \ldots, 0)$. For such $c$, if $\left(w_{1}^{\prime}, \ldots, w_{n}^{\prime}\right)=$ $\left(c_{1}-c, \ldots, c_{n}-c\right)$, then $1 \in F_{0}\left(w_{1}, \ldots, w_{n}\right)$ because $F$ is weakly efficient and satisfies IPM or monotonicity. Similarly, for $c$ sufficiently large, $\left(w_{1}^{\prime}, \ldots, w_{n}^{\prime}\right)$ 
$=\left(c_{1}-c, \ldots, c_{n}-c\right)$ implies that $0 \in F_{0}\left(w_{1}, \ldots, w_{n}\right)$. By the convex-valuedness of $F_{0}$, there exists $c$ satisfying (3). Therefore $h$ is well defined. By Lemma $2, h$ is semi-strictly increasing. Clearly, $h$ is continuous because $F_{0}$ is upper hemicontinuous. If $a>0$, then $h(a, \ldots, a)>0$, because $F_{0}$ is weakly efficient. Similarly, if $a<0, h(a, \ldots, a)<0$. Therefore, by continuity,

$$
h(0, \ldots, 0)=0 \text {. }
$$

Lemma 3. Suppose that $F$ satisfies the hypotheses of Lemma 2. Then, if $\left(v_{1}, \ldots, v_{n}\right) \in V^{n}$,

$$
F_{0}\left(v_{1}, \ldots, v_{n}\right)=\left\{K \mid h\left(v_{1}^{\prime}(K), \ldots, v_{n}^{\prime}(K)\right)=0\right\} .
$$

Proof. By definition of $V, v_{i}^{\prime}(0)>0$ and $v_{i}^{\prime}(1)<0$ for any $v_{i} \in V$. Therefore, because $h$ is semi-strictly increasing and because of $(4), h\left(v_{1}^{\prime}(0), \ldots, v_{n}^{\prime}(0)\right)>0$ and $h\left(v_{1}^{\prime}(1), \ldots, v_{n}^{\prime}(1)\right)<0$. From continuity, the semi-strict monotonicity of $h$, and the strict concavity of the $v_{i}$ 's, we conclude that $\left\{K \mid h\left(v_{1}^{\prime}(K), \ldots, v_{n}^{\prime}(K)\right)=0\right\}$ consists of a single element $K^{*}$. By definition of $h$, there exists $\left(w_{1}, \ldots, w_{n}\right) \in L^{n}$ such that $\frac{1}{2} \in F_{0}\left(w_{1}, \ldots, w_{n}\right)$ and $\left(w_{1}^{\prime}, \ldots, w_{n}^{\prime}\right)=\left(v_{1}^{\prime}\left(K^{*}\right), \ldots, v_{n}^{\prime}\left(K^{*}\right)\right)$. From Lemma 2, $K^{*} \in F_{0}\left(w_{1}, \ldots, w_{n}\right)$. For each $i, v_{i}$ is more concave than $w_{i}$ at $K^{*}$. Therefore, by argument completely paralleling that of Lemma 1 , we conclude that $K^{*} \in F_{0}\left(v_{1}, \ldots, v_{n}\right)$. Because $F_{0}$ is single-valued, $\left\{K^{*}\right\}=F_{0}\left(v_{1}, \ldots, v_{n}\right)$. Q.E.D.

From Lemma 3, we can already conclude that the public decision rules implementable in Nash strategies are the same as those implementable in dominant strategies. We are now ready for the proofs of Theorems 5 and 6 .

Proof of Theorem 5. First suppose that $F$ is implementable in Nash strategies. Consider $\left(K, t_{1}, \ldots, t_{n}\right) \in F\left(v_{1}, \ldots, v_{n}\right)$ for some choice of $\left(v_{1}, \ldots, v_{n}\right) \in V^{n}$. From Lemma 3 , there exist $\left(\tilde{t}_{1}, \ldots, \tilde{t}_{n}\right)$ such that

$$
\left(K, \tilde{t}_{1}, \ldots, \tilde{t}_{n}\right) \in F\left(\tilde{v}_{1}, \ldots, \tilde{v}_{n}\right)
$$

for any $\left(\tilde{v}_{1}, \ldots, \tilde{v}_{n}\right) \in V^{n}$ such that $\tilde{v}_{i}^{\prime}(K)=v_{i}^{\prime}(K)$ for all $i$. Choose $\left(v_{1}^{*}, \ldots, v_{n}^{*}\right) \in V^{n}$ such that for all $i, v_{i}^{*}$ is more concave than either $v_{i}$ or $\tilde{v}_{i}$ at $K$. Then, by previous argument, $\left(K, t_{1}, \ldots, t_{n}\right),\left(K, \tilde{t}_{1}, \ldots, \tilde{t}_{n}\right) \in F\left(v_{1}^{*}, \ldots, v_{n}^{*}\right)$. But $F\left(v_{1}^{*}, \ldots, v_{n}^{*}\right)$ is a singleton. Thus $t_{i}=\tilde{t}_{i}$ for all $i$. We conclude that $t_{i}$ depends only on $K$ and $\left(v_{1}^{\prime}(K), \ldots, v_{n}^{\prime}(K)\right)$, and so we can write $t_{i}=t_{i}^{*}\left(K, v_{1}^{\prime}(K), \ldots, v_{n}^{\prime}(K)\right)$.

These observations, together with Lemma 3, establish that Nash implementability implies the existence of functions $h$ and $t_{i}^{*}$ satisfying (i), (ii), and $\left({ }^{*}\right)$. 
Next, assume the existence of these functions. We will show that $F$ defined by $\left(^{*}\right)$ is implementable. By Theorem 3 it suffices to show that $F$ is monotonic. ${ }^{6}$

Suppose that $\left(K, t_{1}, \ldots, t_{n}\right) \in F\left(v_{1}, \ldots, v_{n}\right)$ for some choice of $\left(v_{1}, \ldots, v_{n}\right) \in V^{n}$. Consider $\left(\tilde{v}_{1}, \ldots, \tilde{v}_{n}\right)$ such that for all $\tilde{K}, i$ and $\tilde{t}_{i}$,

$$
v_{i}(K)+t_{i} \geqq v_{i}(\tilde{K})+\tilde{t}_{i} \Rightarrow \tilde{v}_{i}(K)+t_{i} \geqq \tilde{v}_{i}(\tilde{K})+\tilde{t}_{i} .
$$

As $K \in(0,1),(6)$ implies $v_{i}^{\prime}(K)=\tilde{v}_{i}^{\prime}(K)$. Thus by $\left(^{*}\right),\left(K, t_{1}, \ldots, t_{n}\right) \in F\left(\tilde{v}_{1}, \ldots, \tilde{v}_{n}\right)$. Thus $F$ is monotonic. That $F_{0}$ is upper hemicontinuous follows from $\left({ }^{*}\right)$ and the continuity of $h$. That $F_{0}$ is weakly efficient follows from $\left(^{*}\right)$ and the assumption that $h(0, \ldots, 0)=0 . F_{0}$ is clearly neutral. Finally, $F$ is single-valued on $V^{n}$ from the strict concavity of the $v_{i}^{\prime \prime}$ s and the semi-strict monotonicity of $h$. Q.E.D.

Proof of Theorem 6. First suppose that $F$ is truthfully implementable in dominant strategies. Assume that $\left(K, t_{1}, \ldots, t_{n}\right) \in F\left(v_{1}, \ldots, v_{n}\right)$ for some choice of $\left(v_{1}, \ldots, v_{n}\right) \in V^{n}$. From Lemma $3, K$ satisfies $h\left(v_{1}^{\prime}(K), \ldots, v_{n}^{\prime}(K)\right)=0$. Now, given the strategies of other agents, any two strategies of agent $i$ that lead to the same public decision must result in the same transfer to him; otherwise, he would never choose the strategy leading to the lower transfer. Hence, if $t_{i}^{* *}$ is $i$ 's transfer as a function of $\left(v_{1}, \ldots, v_{n}\right), t_{i}^{* *}$ depends on $v_{i}$ only through $K$, and we may write

$$
t_{i}^{* *}=t_{i}^{* *}\left(K, v_{-i}\right) \text {. }
$$

Take $h_{i}: \boldsymbol{R}^{n-1} \rightarrow \boldsymbol{R}$ as in formula $\left({ }^{* * *}\right)$ of Theorem 6 . Because $v_{-i}^{\prime}(K)$ is decreasing in $K$ and $h$ is semi-strictly increasing, there exist $K_{1}$ and $K_{2}$ with $0 \leqq K_{1} \leqq K_{2} \leqq 1$ such that $h_{i}\left(v_{-i}^{\prime}(K)\right)$ is non-decreasing if $K \in\left[K_{1}, K_{2}\right]$ and equal to zero otherwise (see the definition of $h_{i}$ ). Thus $h_{i}$ is continuous almost everywhere, and so $\int_{0}^{K} h_{i}\left(v_{-i}^{\prime}(t)\right) \mathrm{d} t$ is well defined.

Write

$$
t_{i}^{* *}\left(\tilde{K}, v_{-i}\right)=-\int_{0}^{K} h_{i}\left(v_{-i}^{\prime}(t)\right) \mathrm{d} t+H_{i}\left(\tilde{K}, v_{-i}\right)
$$

We must show that $H_{i}$ is independent of $\tilde{K}$. Suppose that for any $\tilde{v}_{i}, F_{0}\left(\tilde{v}_{i}, v_{-i}\right)$ $=\{K\}$. Then we clearly can drop $\tilde{K}$ in $H_{i}$ since $\tilde{K}$ can only be equal to $K$. Thus suppose there exist $\bar{v}_{i}$ and $\bar{K} \neq K$ such that $\bar{K} \in F_{0}\left(\bar{v}_{i}, v_{-i}\right)$. Without loss of generality, assume $\bar{K}>K$. Because $h$ is continuous, for all $\widetilde{K} \in[K, \bar{K}]$ there exists $\tilde{v}_{i}$ such that $\tilde{K} \in F_{0}\left(\tilde{v}_{i}, v_{-i}\right)$. From (7), we have for $0<\varepsilon<\bar{K}-K$,

$$
\begin{aligned}
& v_{i}(K)-\int_{0}^{K} h_{i}\left(v_{-i}^{\prime}(t)\right) \mathrm{d} t+H_{i}\left(K, v_{-i}\right) \\
& \geqq v_{i}(K+\varepsilon)-\int_{0}^{K+\varepsilon} h_{i}\left(v_{-i}^{\prime}(t)\right) \mathrm{d} t+H_{i}\left(K+\varepsilon, v_{-i}\right) .
\end{aligned}
$$

\footnotetext{
${ }^{6}$ In this framework it suffices to show that $F$ is monotonic.
} 
Hence

$$
v_{i}(K)-v_{i}(K+\varepsilon)+\int_{K}^{K+\varepsilon} h_{i}\left(v_{-i}^{\prime}(t)\right) \mathrm{d} t \geqq H_{i}\left(K+\varepsilon, v_{-i}\right)-H_{i}\left(K, v_{-i}\right) .
$$

Dividing by $\varepsilon$ and letting $\varepsilon$ tend to zero $(\varepsilon>0)$, we obtain

$$
-v_{i}^{\prime}(K)+\lim _{\varepsilon \rightarrow 0} h_{i}\left(v_{-i}^{\prime}(K+\varepsilon)\right) \geqq \limsup _{\varepsilon \rightarrow 0}\left(H_{i}\left(K+\varepsilon, v_{-i}\right)-H_{i}\left(K, v_{-i}\right)\right) / \varepsilon .
$$

Choose $\hat{v}_{i} \in V$ such that

$$
\hat{v}_{i}^{\prime}(K)=\lim _{\varepsilon \rightarrow 0} h_{i}\left(v_{-i}^{\prime}(K+\varepsilon)\right)
$$

Then, because $h$ is continuous $K \in F_{0}\left(\hat{v}_{i}, v_{-i}\right)$. From (9),

$$
\begin{aligned}
& -\hat{v}_{i}^{\prime}(K)+\lim _{\varepsilon \rightarrow 0} h_{i}\left(v_{-i}^{\prime}(K+\varepsilon)\right)=0 \\
& \geqq \limsup _{\varepsilon \rightarrow 0}\left(H_{i}\left(K+\varepsilon, v_{-i}\right)-H_{i}\left(K, v_{-i}\right)\right) / \varepsilon .
\end{aligned}
$$

For each $\varepsilon \in(0, \bar{K}-K)$, take

$$
v_{i}^{\varepsilon}(\tilde{K})=v_{i}(\tilde{K})+\left[h_{i}\left(v_{-i}^{\prime}(K+\varepsilon)\right)-v_{i}^{\prime}(K+\varepsilon)\right] \tilde{K}
$$

Notice that $\left(v_{i}^{\varepsilon}\right)^{\prime}(K+\varepsilon)=h_{i}\left(v_{-i}^{\prime}(K+\varepsilon)\right)$. Thus $K+\varepsilon \in F_{0}\left(v_{i}^{\varepsilon}, v_{-i}\right)$. Hence

$$
\begin{aligned}
& v_{i}^{\varepsilon}(K+\varepsilon)-\int_{0}^{K+\varepsilon} h_{i}\left(v_{-i}^{\prime}(t)\right) \mathrm{d} t+H_{i}\left(K+\varepsilon, v_{-i}\right) \\
& \geqq v_{i}^{\varepsilon}(K)-\int_{0}^{K} h_{i}\left(v_{-i}^{\prime}(t)\right) \mathrm{d} t+H_{i}\left(K, v_{-i}\right) .
\end{aligned}
$$

Rearranging, we obtain

$$
-\left[v_{i}^{\varepsilon}(K+\varepsilon)-v_{i}^{\varepsilon}(K)\right]+\int_{K}^{K+\varepsilon} h_{i}\left(v_{-i}(t)\right) \mathrm{d} t \leqq H_{i}\left(K+\varepsilon, v_{-i}\right)-H_{i}\left(K, v_{-i}\right) .
$$

Dividing by $\varepsilon$ and letting $\varepsilon$ tend to zero, we have

$$
\begin{aligned}
& -v_{i}^{\prime}(K)-\lim _{\varepsilon \rightarrow 0} h_{i}\left(v_{-i}^{\prime}(K+\varepsilon)+v_{i}^{\prime}(K)+\lim _{\varepsilon \rightarrow 0} h_{i}\left(v_{-i}^{\prime}(K+\varepsilon)\right)=0\right. \\
& \leqq \limsup _{\varepsilon \rightarrow 0} H_{i}\left(K+\varepsilon, v_{-i}\right) .
\end{aligned}
$$


Combining (10) and (11), we obtain

Similarly,

$$
\limsup _{\varepsilon \rightarrow 0}\left(H_{i}\left(K+\varepsilon, v_{-i}\right)-H_{i}\left(K, v_{-i}\right)\right) / \varepsilon=0 .
$$

$$
\liminf _{\varepsilon \rightarrow 0}\left(H_{i}\left(K+\varepsilon, v_{-i}\right)-H_{i}\left(K, v_{-i}\right)\right) / \varepsilon=0 .
$$

Thus,

$$
\frac{\partial^{+}}{\partial K} H_{i}\left(K, v_{-i}\right)=0
$$

Therefore $H_{i}$ does not depend on $K$, as was to be shown. This observation together with Lemma 3 establishes that truthful dominant strategy implementation implies the existence of functions $h, H_{i}$ and $t_{i}^{* *}$ satisfying (iii), (iv) and $\left({ }^{* *}\right)$.

Next assume the existence of $h, H_{i}$ and $t_{i}^{* *}$. We will show that $F$ defined by $\left({ }^{* *}\right)$ is implementable. One way to do this is to verify that $F$ satisfies IPM and apply Theorem 1. A more direct way is to observe that the right and left derivatives of

$$
v_{i}(\tilde{K})-\int_{0}^{\tilde{K}} h_{i}\left(v_{-i}^{\prime}(t)\right) \mathrm{d} t+H_{i}\left(v_{-i}\right)
$$

with respect to $\widetilde{K}$, are

$$
v_{i}^{\prime}(\tilde{K})-\lim _{\varepsilon \rightarrow 0} h_{i}\left(v_{-i}^{\prime}(\tilde{K}+\varepsilon)\right), \quad \varepsilon>0
$$

and

$$
v_{i}^{\prime}(\tilde{K})-\lim _{\varepsilon \rightarrow 0} h_{i}\left(v_{-i}^{\prime}(\tilde{K}-\varepsilon)\right), \quad \varepsilon>0 .
$$

Now, for $v_{i} \in V,(13)$ and (14) are both decreasing in $\tilde{K}$. Therefore, (12) is a strictly concave function of $\widetilde{K}$. Furthermore if $K$ satisfies $h\left(v_{i}^{\prime}(K), \ldots, v_{n}^{\prime}(K)\right)=0$, then $(13)$ is non-positive and (14) is non-negative. Thus $K$ maximizes (12) and so truthfulness in a dominant strategy.

Finally suppose that $h$ is strictly increasing. We must verify that $F$ is strictly truthfully implementable. First observe that because $h$ is strictly increasing, there exists $v_{-i} \in V^{n-1}$ such that $K \in F_{0}\left(v_{i}, v_{-i}\right)$ if $v_{i}^{\prime}(K)$ is sufficiently close to zero [recall that $h(0, \ldots, 0)=0]$. Hence if $v_{i}^{\prime} \neq \tilde{v}_{i}^{\prime}$, we can choose $K$ and $v_{-i}$ such that $v_{i}^{\prime}(K) \neq \tilde{v}_{i}^{\prime}(K)$ and $K \in F_{0}\left(v_{i}, v_{-i}\right)$, establishing condition (i) of Theorem 2. For given $v_{-i} \in V^{n-1}$,

$$
\begin{aligned}
& \left\{\left(F_{0}\left(v_{i}, v_{-i}\right), F_{i}\left(v_{i}, v_{-i}\right)\right) \backslash v_{i} \in V\right\} \\
& =\left\{\left(K, t_{i}\right) \backslash h\left(v_{i}^{\prime}(K), v_{-i}^{\prime}(K)\right)=0, t_{i}=-\int_{0}^{K} h_{i}\left(v_{-i}^{\prime}(t)\right) \mathrm{d} t+H_{i}\left(v_{-i}\right), v_{i} \in V\right\}
\end{aligned}
$$


But, because $h$ is strictly increasing, the right-hand side of (15) defines a differentiable curve. ${ }^{7}$ Thus condition (ii) of Theorem 2 holds, and the theorem is proved. Q.E.D.

Theorems 5 and 6 can easily be generalized to a public project space consisting of the $m$-dimensional unit cube $[0,1]^{m}$. Define $V, L, V^{*}$ and the terms 'weakly efficient' and 'neutral' by analogy with the one-dimensional case. Theorem 5 reads exactly as before except that $h$ is now defined on $\boldsymbol{R}^{m n}$ and the $t_{i}$ 's are defined on $[0,1]^{m} \times \boldsymbol{R}^{m n}$. Similarly, Theorem 6 remains exactly the same except that the $h_{i}$ 's arc defincd on $\boldsymbol{R}^{m(n-1)}$.

Theorems 5 and 6 show that a Nash or dominant strategy implementable public decision rule corresponds to the set of roots of a continuous function that is semi-strictly increasing and satisfies $h(0, \ldots, 0)=0$. Thus for the case of two individuals it is easy to describe implementable public decision rules by the locus of roots of the corresponding $h$ functions. Any implementable social choice rule must have an $h$ whose locus of roots lies between those of $h_{\min }$ and $h_{\max }$ which are depicted in figs. 3 and 4 ,

$$
\begin{aligned}
& h_{\min }\left(x_{1}, x_{2}\right)=\min \left\{x_{1}, x_{2}\right\}, \\
& h_{\max }\left(x_{1}, x_{2}\right)=\max \left\{x_{1}, x_{2}\right\} .
\end{aligned}
$$

The decision rule associated with $h_{\min }$ is the dictatorship of the 'agent on the left', i.e., the public decision coincides with the smaller of the two agents' favorite choices. Similarly $h_{\max }$ corresponds to the dictatorship of 'the agent on the right'. For $n>2$, we can also implement dictatorships of all other 'positions' (e.g. dictatorship of the median agent - majority rule).

For a positional dictatorship $\int_{0}^{K} h_{i}\left(v_{-i}^{\prime}(t)\right) \mathrm{d} t=0$ for any $i$, and therefore we can implement such dictatorships in dominant strategies with transfers that are independent of the public decision. In particular, we can take them to be identically zero, putting us in the [Moulin (1980)] framework of single-peaked preferences over a single good.

Fig. 5 depicts the dictatorship of agent 1 and fig. 6 the dictatorship of agent 2; in both cases transfers are independent of the public decision, as $\int_{0}^{K} h_{i}\left(v_{-i}^{\prime}(t)\right) \mathrm{d} t \equiv 0$.

The social choice rule corresponding to the $h$ function in fig. 7 is a dictatorship by agent 2 as long as agent 1's marginal valuation at agent 2's favorite choice lies between $\alpha$ and $\beta$. In this case transfers are independent of the public decision. For marginal valuations by agent 1 outside that range, the social choice rule chooses $K$ to maximize $v_{1}+v_{2}$ (the 'utilitarian' rule) and transfers of the Clarke-Groves form.

\footnotetext{
${ }^{7} \mathrm{We}$ are implicitly assuming here that $H_{i}$ is differentiable. This is justified because adding an arbitrary function of $v_{-i}$ to $H_{i}$ does not affect incentives.
} 


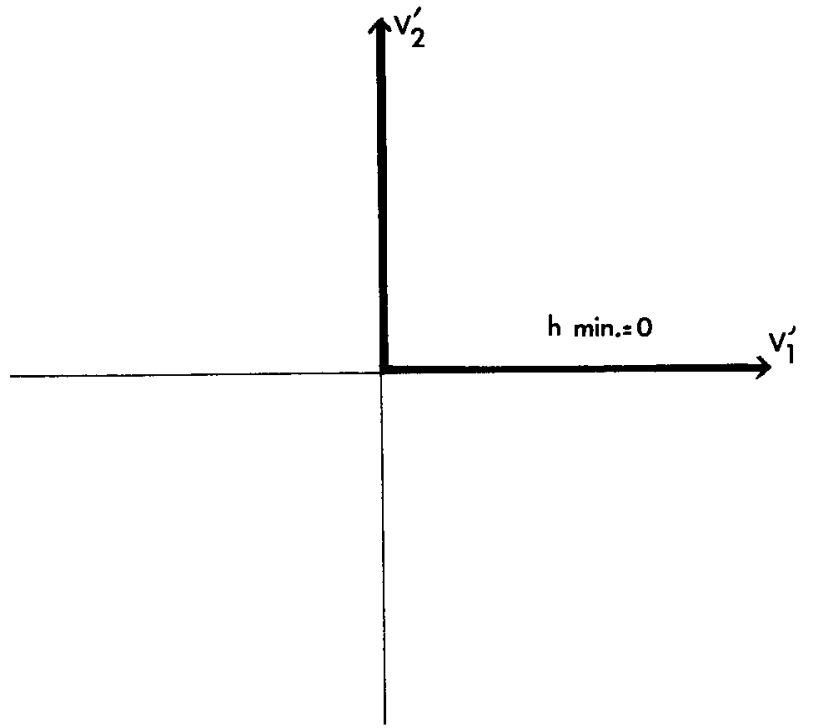

Fig. 3

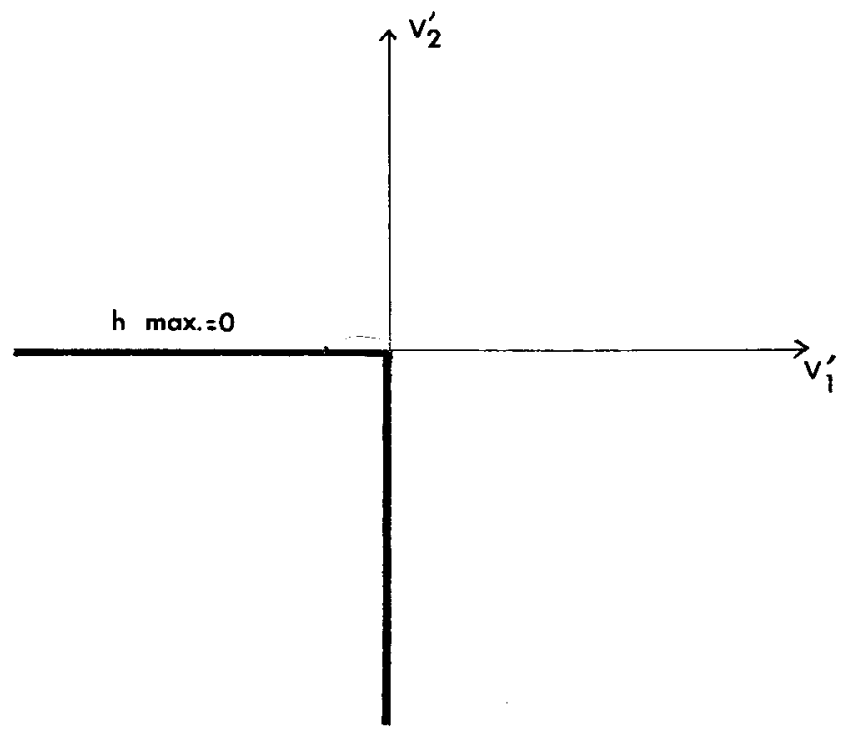

Fig. 4 


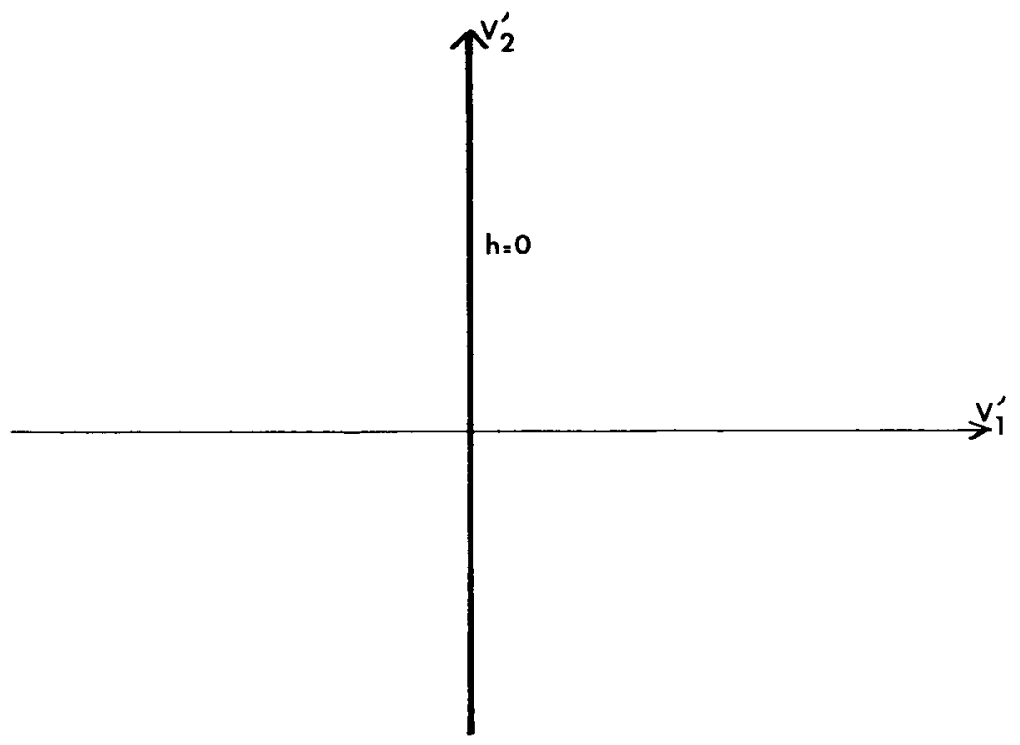

Fig. 5

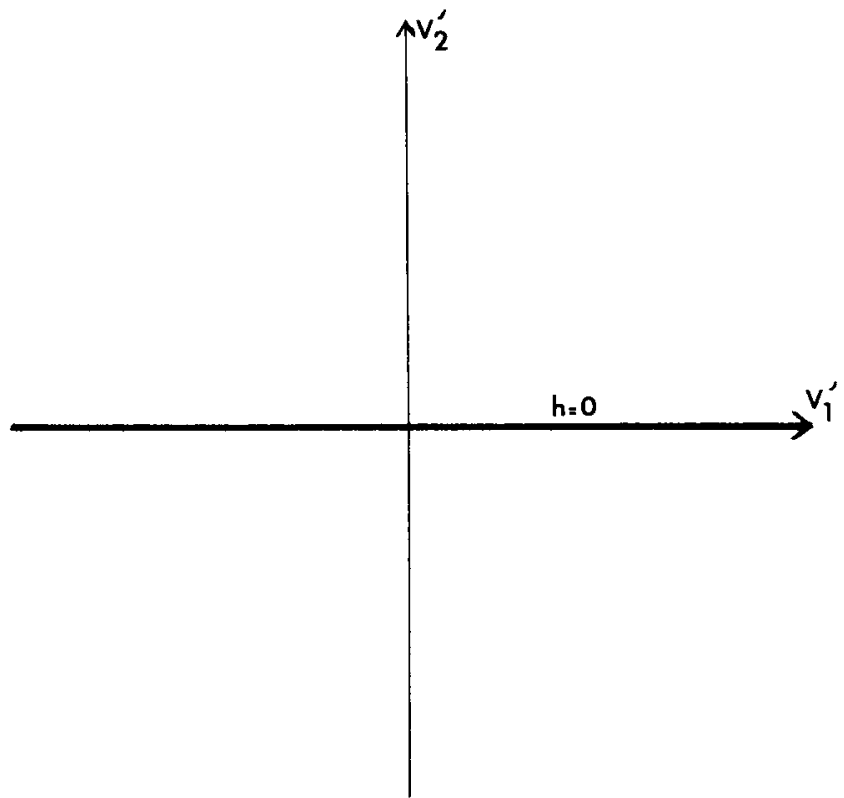

Fig. 6 


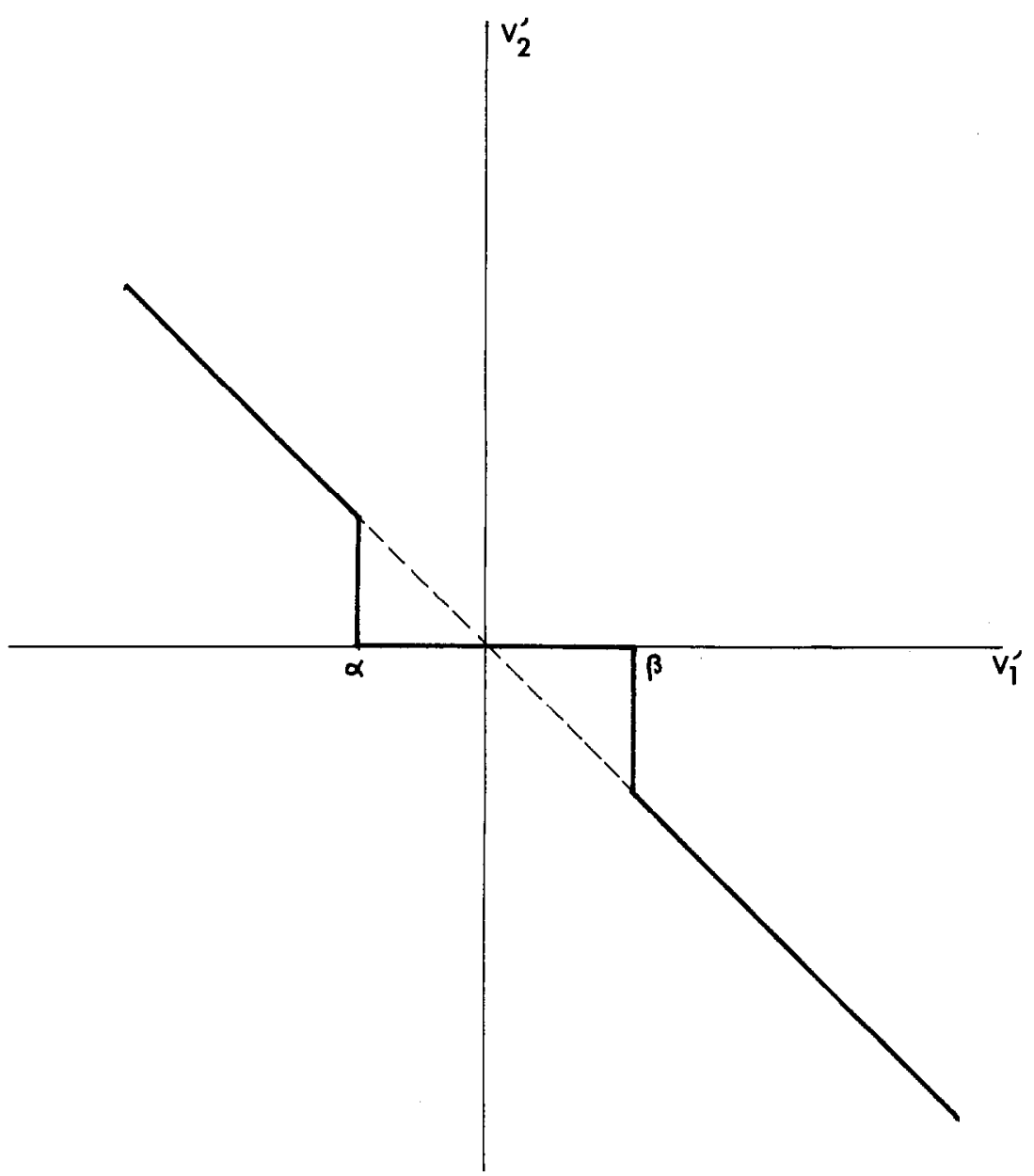

Fig. 7

In fig. 8 we represent a social choice rule that is a dictatorship by agent 2 if agent l's marginal valuation is sufficiently close to zero and otherwise a dictatorship by agent 1 . Fig. 9 depicts a social choice rule that is a dictatorship by agent 2 as long as $v_{i}^{\prime}$ is positive, i.e., as long as agent 2 's favorite public decision is smaller than that of agent 1 , and the utilitarian rule otherwise.

\section{Coalitions}

Theorem 6 concerns implementation only in individually dominant strategies. That is, it ignores the possibility of collusion by coalitions. Returning to the abstract framework of section 2, we say that a direct revelation mechanism 


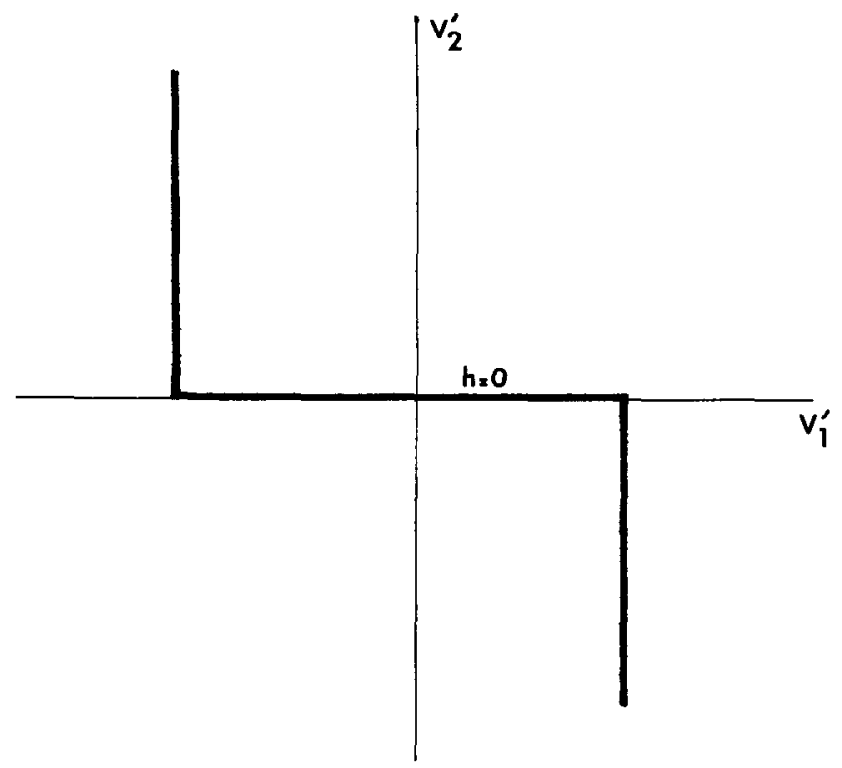

Fig. 8

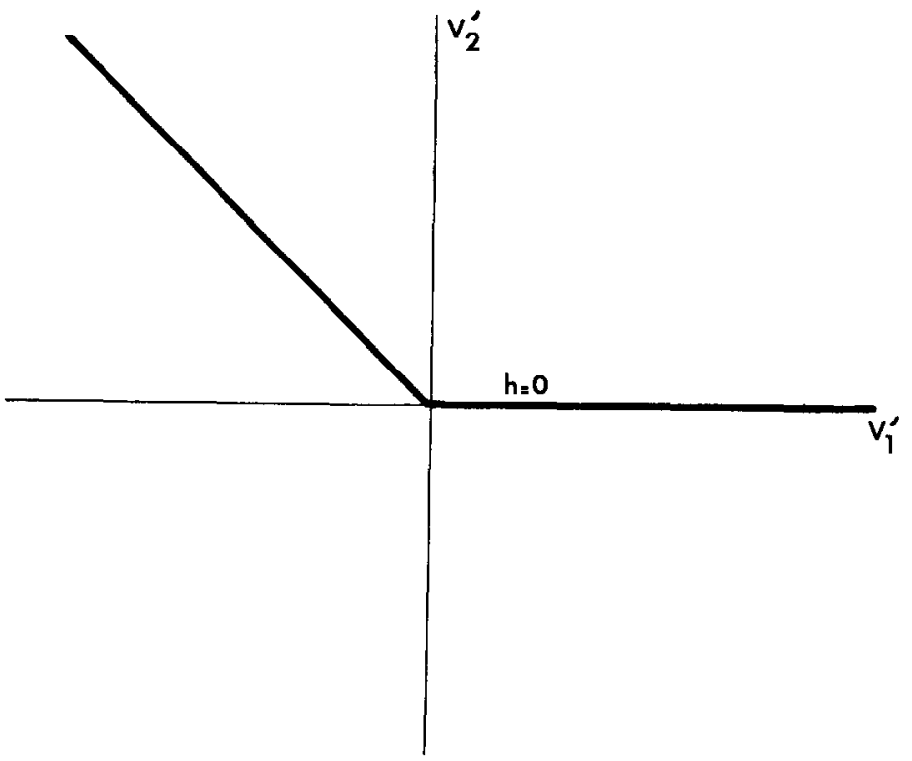

Fig. 9 
$g: \prod_{i=1}^{n} \mathscr{R}_{i} \rightarrow X$ (partially) implements a social choice rule $F$ truthfully in coalitionally dominant strategies if for all $\left(R_{1}, \ldots, R_{n}\right) \in \prod_{i=1}^{n} \mathscr{R}_{i}$ and all coalitions $C \subseteq\{1, \ldots, n\}, \quad g\left(R_{1}, \ldots, R_{n}\right) \in F\left(R_{1}, \ldots, R_{n}\right)$ and the joint strategy $R_{C}=$ $\left(R_{i_{1}}, \ldots, R_{i_{m}}\right)$, where $C=\left\{i_{1}, \ldots, i_{m}\right\}$, is a coalitionally dominant strategy for coalition $C$ with preferences $R_{C}$ [that is, for all alternative $\tilde{R}_{C}$ $g\left(R_{C}, R_{-C}\right) R_{i} g\left(\tilde{R}_{C}, R_{-C}\right)$ for all $i$ in $C$ where $R_{-C}$ is the vector of strategies of agents not in $C$ ]. $F$ is truthfully implementable in coalitionally dominant strategies if $\forall\left(R_{1}, \ldots, R_{n}\right) \in \prod_{i=1}^{n} \mathscr{R}_{i}, \forall a \in f\left(R_{1}, \ldots, R_{n}\right)$, there exists $g$ such that $g$ partially implements $F$ truthfully in coalitionally dominant strategies and $g\left(R_{1}, \ldots, R_{n}\right)=a$. Under the hypotheses of Theorem 6, however, truthful implementation in coalitionally dominant strategies is not attainable.

Theorem 7. If F satisfies the hypotheses of Theorem 6 and is twice piecewise differentiable, ${ }^{8}$ then it is not truthfully implementable in coalitionally dominant strategies.

Proof. Let us confine our attention to the subclass of valuations of the form

$$
v_{i}\left(K, \alpha_{i}, \beta_{i}\right)=\alpha_{i} K-\beta_{i} K^{2}, \quad \alpha_{i}>0, \quad \beta_{i}>0 .
$$

Then for $\left(K, t_{1}, \ldots, t_{n}\right) \in F\left(v_{1}, \ldots, v_{n}\right)$,

$$
\begin{aligned}
& K=K\left(\alpha_{1}, \beta_{1}, \ldots, \alpha_{n}, \beta_{n}\right) \equiv K(\alpha, \beta), \\
& t_{i}=t_{i}\left(\alpha_{1}, \beta_{1}, \ldots, \alpha_{n}, \beta_{n}\right) \equiv t_{i}(\alpha, \beta),
\end{aligned}
$$

and we can regard $F$ as a function of $(\alpha, \beta)$. Choose $j$ and $(\hat{\alpha}, \hat{\beta})$ such that $F$ is twice differentiable at $(\hat{\alpha}, \hat{\beta})$ and $\left(\partial K / \partial \alpha_{j}\right)(\hat{\alpha}, \hat{\beta}) \neq 0$. Then,

$$
\frac{\partial^{2} t_{i}}{\partial \alpha_{i} \partial \alpha_{j}} \equiv \begin{gathered}
\partial^{2} t_{i} \\
\partial \alpha_{j} \partial \alpha_{i}
\end{gathered}
$$

If $F$ is implementable coalitionally, it is implementable individually. Hence,

and so

$$
\left.\frac{\partial}{\partial \alpha_{i}}\left[v_{i}\left(K(\alpha, \beta), \hat{\alpha}_{i}, \hat{\beta}_{i}\right)+t_{i}(\alpha, \beta)\right]\right|_{\left(\alpha_{i}, \beta_{i}\right)=\left(\hat{\alpha}_{i}, \hat{\beta}_{i}\right)}=0,
$$

$$
\frac{\partial t_{i}}{\partial \alpha_{i}}=-\frac{\partial v_{i}}{\partial K} \cdot \frac{\partial K}{\partial \alpha_{i}}
$$

The coalition $\{i, j\}$ with parameters $\left(\hat{\alpha}_{i}, \hat{\beta}_{i}\right)$ and $\left(\alpha_{j}, \beta_{j}\right)$ chooses $\alpha_{i}$ to maximize

$$
v_{i}\left(K(\alpha, \beta), \hat{\alpha}_{i}, \hat{\beta}_{i}\right)+v_{j}\left(K(\alpha, \beta), \hat{\alpha}_{j}, \hat{\beta}_{j}\right)+t_{i}(\alpha, \beta)+t_{j}(\alpha, \beta) .
$$

${ }^{8}$ Henceforth we consider single-valued $F$ 's restricted to $V^{n}$. 
Hence,

$$
\frac{\partial v_{i}}{\partial K} \cdot \frac{\partial K}{\partial \alpha_{i}}+\frac{\partial v_{j}}{\partial K} \cdot \frac{\partial K}{\partial \alpha_{i}}+\frac{\partial t_{i}}{\partial \alpha_{i}}+\frac{\partial t_{j}}{\partial \alpha_{i}}=0
$$

Subtracting (19) from (20) we obtain

$$
\frac{\partial t_{j}}{\partial \alpha_{i}}=-\frac{\partial v_{j}}{\partial K} \cdot \frac{\partial K}{\partial \alpha_{i}}
$$

Interchanging $i$ and $j$, we have

$$
\frac{\partial t_{i}}{\partial \alpha_{j}}=-\frac{\partial v_{i}}{\partial K} \cdot \frac{\partial K}{\partial \alpha_{j}} .
$$

From (18), (19) and (21), we deduce

$$
\frac{\partial^{2} v_{i}}{\partial K \partial \alpha_{i}} \cdot \frac{\partial K}{\partial \alpha_{j}}=0
$$

From (16), $\partial^{2} v_{i} / \partial K \partial \alpha_{i}=1$. Therefore (22) implies that $\partial K / \partial \alpha_{j}=0$, which contradicts $(17)$ for $(\alpha, \beta)=(\hat{\alpha}, \hat{\beta})$. Q.E.D.

\section{Balance}

We know from Theorem 5 that for any Nash implementable public decision rule, balanced transfers (i.e., transfers which sum to zero) can be found that make the resulting social choice rule Nash implementable. For example, transfers can be taken to be identically zero. On the other hand, Green and Laffont (1979) have shown that no social rule that is implementable in dominant strategies and has a utilitarian public decision rule (i.e., chooses $K$ to maximize $\sum_{i} v_{i}$ ) has transfers which balance. This non-existence result does not carry over to all nonutilitarian public decision rules. For instance, we observed already that any dictatorship or positional dictatorship without transfers is implementable. Such social choice rules are automatically balanced. As another example, consider the social choice rule

$$
\begin{aligned}
F^{*}\left(v_{1}, \ldots, v_{n}\right)= & \left\{\left(K, t_{1}, \ldots, t_{n}\right) \sum_{i=1}^{n-1} v_{i}^{\prime}(K)=0,\right. \\
& \left.t_{i}=\sum_{j \neq i, n} v_{j}(K), i \neq n, \quad t_{n}=-\sum_{i=1}^{n-1} t_{i}\right\} .
\end{aligned}
$$


Clearly, $F^{*}$ is balanced and implementable. Notice that balance is achieved by making agent $n$ a dummy. That is, agent $n$ cannot affect the public decision $K$ and serves simply to absorb the surplus or deficit created by the other agents. $F^{*}$ treats agents highly asymmetrically and there are many other balanced and implementable social choice rules that entail such asymmetries. However, if one restricts attention to symmetric - i.e., anonymous - social choice rules, we find that only the positional dictatorships are balanced and implementable.

We shall call a social choice rule anonymous if permutating the valuation functions in a profile $\left(v_{1}, \ldots, v_{n}\right)$ permutes transfers the same way and does not affect the public decision.

Theorem 8. Suppose that $F$ satisfies the hypotheses of Theorem 6 and is $n$ times piecewise differentiable, balanced and anonymous; then, $F$ is truthfully implementable in dominant strategies if and only if $F$ is a positional dictatorship.

Proof. For simplicity, we shall argue the case $n=2$; the method of argument generalizes. We shall restrict attention to the class of valuation functions

$$
v_{i}\left(K, \alpha_{i}, \beta_{i}, \theta_{i}\right)=\alpha_{i} K-\beta_{i} K^{2}+\theta_{i} K^{3}, \quad \alpha_{i}, \beta_{i}>0
$$

Thus we may consider $F$ as a function (since it is single-valued) of $(\alpha, \beta, \theta)$ $=\left(\alpha_{1}, \beta_{1}, \theta_{1}, \alpha_{2}, \beta_{2}, \theta_{2}\right)$.

In view of Theorem 6 , balance requires that

$$
\begin{aligned}
\int_{0}^{K(\alpha, \beta, \theta)} & -\left(h_{1}\left(\frac{\partial v_{2}}{\partial K}\left(t, \alpha_{2}, \beta_{2}, \theta_{2}\right)\right)+h_{2}\left(\frac{\partial v_{1}}{\partial K}\left(t, \alpha_{1}, \beta_{1}, \theta_{1}\right)\right)\right) d t \\
& +H_{1}\left(\alpha_{2}, \beta_{2}, \theta_{2}\right)+H_{2}\left(\alpha_{1}, \beta_{1}, \theta_{1}\right)=0 .
\end{aligned}
$$

Differentiating (23) with respect to $\alpha_{1}$ and $\alpha_{2}$, we obtain

$$
\begin{aligned}
& \left(h_{1}+h_{2}\right) \frac{\partial^{2} K}{\partial \alpha_{1} \partial \alpha_{2}}+\frac{\partial K}{\partial \alpha_{1}} \cdot \frac{\partial h_{1}}{\partial v_{2}^{\prime}}+\frac{\partial K}{\partial \alpha_{2}} \cdot \frac{\partial h_{2}}{\partial v_{1}^{\prime}} \\
& +\frac{\partial K}{\partial \alpha_{1}} \cdot \frac{\partial K}{\partial \alpha_{2}}\left[\frac{\partial h_{1}}{\partial v_{2}^{\prime}} \cdot \frac{\partial^{2} v_{2}}{\partial K^{2}}+\frac{\partial h_{2}}{\partial v_{1}^{\prime}} \cdot \frac{\partial^{2} v_{1}}{\partial K^{2}}\right]=0 .
\end{aligned}
$$

Also from Thcorem 6 we have

$$
h\left(\frac{\partial v_{1}}{\partial K}, \frac{\partial v_{2}}{\partial K}\right)=0
$$


Differentiating (25) with respect to $\alpha_{1}$ and $\alpha_{2}$, we obtain

$$
\left(\frac{\partial h}{\partial v_{1}^{\prime}} \frac{\partial^{2} v_{1}}{\partial K^{2}}+\frac{\partial h}{\partial v_{2}^{\prime}} \frac{\partial^{2} v_{2}}{\partial K^{2}}\right) \frac{\partial K}{\partial \alpha_{i}}+\frac{\partial h}{\partial v_{i}^{\prime}}=0,
$$

and

$$
\begin{aligned}
& \left(\frac{\partial h}{\partial v_{1}^{\prime}} \frac{\partial^{2} v_{1}}{\partial K^{2}}+\frac{\partial h}{\partial v_{2}^{\prime}} \frac{\partial^{2} v_{2}}{\partial K^{2}}\right) \frac{\partial^{2} K}{\partial \alpha_{1} \partial \alpha_{2}}+\frac{\partial K}{\partial \alpha_{1}} \frac{\partial K}{\partial \alpha_{2}}\left(\frac{\partial h}{\partial v_{1}^{\prime}} \frac{\partial^{3} v_{1}}{\partial K^{3}}+\frac{\partial h}{\partial v_{2}^{\prime}} \frac{\partial^{3} v_{2}}{\partial K^{3}}\right) \\
& +\left\{\text { terms of lower order derivatives of } v_{i} \text { and } K\right\}=0 .
\end{aligned}
$$

Choose $(\bar{\alpha}, \bar{\beta}, \bar{\theta})$ where $F$ is differentiable and such that

$$
\left.\frac{\partial h}{\partial v_{1}^{\prime}}\right|_{\bar{\alpha}, \bar{\beta}, \bar{\theta}} \neq 0 \neq\left.\frac{\partial h}{\partial v_{2}^{\prime}}\right|_{\bar{\alpha}, \bar{\beta}, \bar{\theta}} .
$$

Substituting (26) and (27) in (24), we obtain

$$
\begin{aligned}
& -\left(\left(h_{1}+h_{2}\right)\left(\frac{\partial h}{\partial v_{1}^{\prime}} \frac{\partial^{3} v_{1}}{\partial K^{3}}+\frac{\partial h}{\partial v_{2}^{\prime}} \frac{\partial^{3} v_{2}}{\partial K^{3}}\right) /\left(\frac{\partial h}{\partial v_{1}^{\prime}} \frac{\partial^{2} v_{1}}{\partial K^{2}}+\frac{\partial h}{\partial v_{2}^{\prime}} \frac{\partial^{2} v_{2}}{\partial K^{2}}\right)^{3}\right) \\
& \times \frac{\partial h}{\partial v_{1}^{\prime}} \frac{\partial h}{\partial v_{2}^{\prime}}+\left\{\text { terms not involving } \frac{\partial^{3} v_{i}}{\partial K^{3}}\right\}=0 .
\end{aligned}
$$

Now, suppose (29) is evaluated at $(\bar{\alpha}, \bar{\beta}, \bar{\theta})$. If $\theta_{1}$ and $\theta_{2}$ vary starting from $\left(\bar{\theta}_{1}, \bar{\theta}_{2}\right)$ we can adjust $\alpha$ and $\beta$ to keep $\partial v_{i} / \partial K$ and $\partial^{2} v_{i} / \partial K^{2}$ constant. Therefore, (29) cannot hold in a neighborhood of $(\bar{\alpha}, \bar{\beta}, \bar{\theta})$ unless

$$
h_{1}+h_{2}=0 \text { at }(\bar{\alpha}, \bar{\beta}, \bar{\theta}) .
$$

But if (30) holds at cach point $(\bar{\alpha}, \bar{\beta}, \bar{\theta})$ where $F$ is differentiable and (28) holds, then in a neighborhood of $(\bar{\alpha}, \bar{\beta}, \bar{\theta}) F$ must be a Clarke-Groves procedure, i.e., locally $h\left(a_{1}, a_{2}\right)=a_{1}+a_{2}$. From Corollary 4.1 in Laffont and Maskin (1980), ClarkeGroves procedures cannot be balanced. Thus we conclude that (28) cannot hold. Consequently the locus $h=0$ must consist entirely of vertical and horizontal segments.

Suppose without loss of generality, that there exists $\bar{a}>0$ such that for all $a \in[0, \bar{a}], h(0, a)=0$; that is, there is a vertical segment of the locus $h=0$ at $(0,0)$. Suppose that $\bar{a}=\max \{a \mid h(0, a)=0\}$. Choose $(\bar{\alpha}, \bar{\beta}, \bar{\theta})$ such that

$$
\left.\left(\frac{\partial v_{1}}{\partial K}, \frac{\partial v_{2}}{\partial K}\right)\right|_{(\bar{\alpha}, \bar{\beta}, \bar{\theta})}=(0, \bar{a})
$$


and let $\bar{K}$ be the associated public decision level. Now, at $\bar{K}$, for $\alpha_{2}>\bar{\alpha}_{2}$,

$$
\left.\frac{\partial v_{2}}{\partial K}\right|_{\left(\alpha_{2}, \bar{\beta}_{2}, \bar{\theta}_{2}\right)}>\left.\frac{\partial v_{2}}{\partial K}\right|_{\left(\bar{\alpha}_{2}, \bar{\beta}_{2}, \bar{\theta}_{2}\right)} .
$$

Therefore, for $\alpha_{2}$ slightly greater than $\bar{\alpha}_{2}$, we must be on the horizontal segment, i.e.,

$$
\left.\left(\frac{\partial v_{1}}{\partial K}, \frac{\partial v_{2}}{\partial K}\right)\right|_{\left(\bar{\alpha}_{1}, \bar{\beta}_{1}, \bar{\theta}_{1}, \alpha_{2}, \bar{\beta}_{2}, \bar{\theta}_{2}\right)}=(-b, \bar{a}) \text { for some } b>0 .
$$

From (32) and Theorem 6,

$$
\begin{aligned}
\frac{\mathrm{d}^{+}}{\mathrm{d} \alpha_{2}}\left(t_{1}+t_{2}\right)= & \frac{\mathrm{d}^{+}}{\mathrm{d} \alpha_{2}}\left(-\int_{0}^{K\left(\bar{\alpha}_{1}, \bar{\beta}_{1}, \bar{\theta}_{1} \cdot \alpha_{2}, \bar{\beta}_{2}, \bar{\theta}_{2}\right)}\left(h_{1}\left(\frac{\partial v_{2}}{\partial K}\right)+h_{2}\left(\frac{\partial v_{1}}{\partial K}\right)\right) \mathrm{d} t\right. \\
& \left.+H_{1}\left(\alpha_{2}, \bar{\beta}_{2}, \bar{\theta}_{2}\right)+H_{2}\left(\bar{\alpha}_{1}, \bar{\beta}_{1}, \bar{\theta}_{1}\right)\right) \\
= & -\left(h_{1}\left(\frac{\partial v_{2}}{\partial K}\right)+h_{2}\left(\frac{\partial v_{1}}{\partial K}\right)\right) \frac{\partial K^{+}}{\partial \alpha_{2}}+\frac{\partial H_{1}^{+}}{\partial \alpha_{2}}
\end{aligned}
$$

where the derivatives are evaluated at $(\bar{\alpha}, \bar{\beta}, \bar{\theta})$.

Now we have

$$
\frac{\partial K^{+}}{\partial \alpha_{2}}>0, \quad h_{1}\left(\frac{\partial v_{2}}{\partial K}\right)=0, \quad h_{2}\left(\frac{\partial v_{1}}{\partial K}\right)=\bar{a} .
$$

Balance implies

$$
\frac{\partial H_{1}^{+}}{\partial \alpha_{2}}=h_{2}\left(\frac{\partial v_{1}}{\partial K}\right) \frac{\partial K^{+}}{\partial \alpha_{2}}>0
$$

On the other hand, the same computation at the point $(0, \bar{a}-\varepsilon)$ (obtained by varying the characteristics of agent 1 ) yields

$$
\frac{\partial H_{1}^{+}}{\partial \alpha_{2}}=0, \text { contradicting balance. }
$$

Therefore, $\max \{a \mid h(0, a)=0\}=+\infty$ and so $h(0, a)=0$ for all $a>0$. By symmetry, $h(a, 0)=0$ for all $a \geqq 0$. Thus $F$ is the 'left' dictatorship. Analogously, we would have obtained the right dictatorship had we assumed that $h(0, a)=0$ for all $a \in[\bar{a}, 0]$ for $\bar{a}<0$. Q.E.D. 


\section{Individual rationality}

A social choice rule is said to be individually rational if, for any profile of preferences, any outcome in the choice set yields each individual as much utility as from his initial position. In our framework, the natural assumption is to suppose that an individual with valuation $v$ derives utility 0 from his initial position. Individual rationality is a desirable feature of a social rule because it ensures that no individual need be coerced into playing an implementing game form; all individuals will play voluntarily.

Because valuation functions are bounded below, it is obvious that any SCR that provides sufficiently large transfers to all agents will be individually rational. However, it is natural to restrict attention to SCR's the sum of whose transfers is always non-positive, that is, to feasible SCR's. As the following result shows, there is only one individually rational and feasible SCR (satisfying the hypotheses of Theorem 6) that is truthfully implementable in dominant strategies.

Theorem 9. Suppose that $F$ is feasible and individually rational and satisfies the hypotheses of Theorem 6. Then $F$ is implementable in dominant strategies if and only if $F$ is the left dictator.

Proof. We shall argue the case of two agents, though our method extends to any number. If $F$ satisfies the hypotheses of Theorem 6 and is truthfully implementable in dominant strategies, then

$$
t_{1}^{* *}\left(v_{1}^{\prime}(K), v_{2}^{\prime}(K)\right)=-\int_{0}^{\kappa} h_{1}\left(v_{2}^{\prime}(t)\right) \mathrm{d} t+H_{1}\left(v_{2}\right)
$$

We shall first show that if $F$ is feasible and individually rational, $H_{1}\left(v_{2}\right) \geqq 0$.

Let $b$ be the smallest non-negative number such that there exists $a$ with $h\left(a, v_{2}^{\prime}(0)-b\right)=0$. Because $h(0,0)=0, b \leqq v_{2}^{\prime}(0)$. Choose $\bar{K}$ such that $v_{2}^{\prime}(\bar{K})=v_{2}^{\prime}(0)$ $-b$. If $b \neq 0$, let $\left\{v_{1}^{n}\right\}$ be a sequence of valuation functions and $\left\{K^{n}\right\}$ an ascending sequence of public decisions such that (i) $K^{n} \rightarrow \bar{K}$, (ii) $\left(v_{1}^{n}\right)^{\prime}\left(K^{n}\right)=h_{1}\left(v_{2}^{\prime}\left(K^{n}\right)\right)$, and (iii) $v_{1}^{n}\left(K^{n}\right) \rightarrow 0$. Bccausc $v_{2}^{\prime}(0)-b \geqq 0, v_{2}^{\prime}\left(K^{n}\right) \geqq 0$, and consequently $h_{1}\left(v_{2}^{\prime}\left(K^{n}\right)\right) \leqq 0$. Then, by construction,

$$
\lim _{n \rightarrow \infty}\left(v_{1}^{n}\left(K^{n}\right)-\int_{0}^{K^{n}} h_{1}\left(v_{2}^{\prime}(t)\right) \mathrm{d} t\right)=0 .
$$

If $b-0$ take a descending sequence $\left\{K^{n}\right\}$ going to $\bar{K}=0$. Thus, by individual rationality, $H_{1}\left(v_{2}\right) \geqq 0$.

Now, suppose there exists an interval $[a, \bar{a}]$ such that for $a \in[a, \bar{a}], h_{1}(a)<0$. Choose $v_{2}$ such that $v_{2}^{\prime}(1 / 2)=0$, and

$$
\bar{a} \geqq v_{2}^{\prime}(K) \geqq \underline{a} \text { for } K<1 / 4 \text {. }
$$


Choose $v_{1}$ such that $v_{1}^{\prime}(1 / 2)=0$. Agent 2's transfer is

$$
-\int_{0}^{1 / 2} h_{2}\left(v_{1}^{\prime}(t)\right) \mathrm{d} t+H_{2}\left(v_{1}\right)
$$

Agent 1's transfer is

$$
-\int_{0}^{1 / 2} h_{1}\left(v_{2}^{\prime}(t)\right) \mathrm{d} t+H_{1}\left(v_{2}\right)
$$

Since $v_{2}^{\prime}(t) \geqq 0$ for $t \in[0,1 / 2], h_{1}\left(v_{2}^{\prime}(t)\right) \leqq 0$ in this interval and from above $h_{1}\left(v_{2}^{\prime}(t)\right)<0$ for $K<1 / 4$.

Hence (38) is strictly positive since $H_{1}\left(v_{2}\right) \geqq 0$.

Since $v_{1}^{\prime}(t) \geqq 0$ for $0 \leqq t \leqq 1 / 2, h_{2}\left(v_{1}^{\prime}(t)\right) \leqq 0$ in this interval and (37) is non-negative from $H_{2}\left(v_{1}\right) \geqq 0$. The sum of the transfers $[(37)+(38)]$ is strictly positive, a violation of feasibility.

Thus $h_{1} \geqq 0$ everywhere and similarly $h_{2} \geqq 0$ everywhere.

Now for any $v_{1}$, choose $v_{2}$ such that for some $K, v_{1}^{\prime}(K)=v_{2}^{\prime}(K)=0$. Then, agent 2 's transfer is

$$
-\int_{0}^{K} h_{2}\left(v_{1}^{\prime}(t)\right) \mathrm{d} t+H_{2}\left(v_{1}\right)
$$

Since $v_{1}^{\prime}(t) \geqq 0$ for any $t$ in $[0, K], h_{2}\left(v_{1}^{\prime}(t)\right) \leqq 0$. Combining with above, we obtain $h_{2}\left(v_{1}^{\prime}(t)=0\right.$ for $t$ in $[0, K]$. Therefore agent 2 's transfer is $H_{2}\left(v_{1}\right)$. Similarly agent 1 's transfer is $H_{1}\left(v_{2}\right)$. From feasibility $H_{1}\left(v_{2}\right)+H_{2}\left(v_{1}\right) \leq 0$. Thus, from the nonnegativity of the $H_{i}$ 's we conclude that

$$
H_{1} \equiv H_{2} \equiv 0
$$

Now suppose there exist $K$ and $\left(v_{1}, v_{2}\right)$ such that $h\left(v_{1}^{\prime}(K), v_{2}^{\prime}(K)\right)=0$ and $v_{1}^{\prime}(K)<0$. Choose $\tilde{v}_{1}$ such that $\tilde{v}_{1}^{\prime}(K)=v_{1}^{\prime}(K)$ and $\tilde{v}_{1}(K)<0$. Then, by Theorem 6 , agent 1 's payoff is

$$
\tilde{v}_{1}(K)-\int_{0}^{K} h_{1}\left(v_{2}^{\prime}(t)\right) \mathrm{d} t+H_{1}\left(v_{2}\right)
$$

But $H_{1}=0$ and $h_{1} \geqq 0$. Therefore, (40) is negative, a contradiction of individual rationality. Thus for all $K$ and $\left(v_{1}, v_{2}\right), h\left(v_{1}^{\prime}(K), v_{2}^{\prime}(K)\right)=0$ only if $v_{1}^{\prime}(K) \geqq 0$ and $v_{2}^{\prime}(K) \geqq 0$. Thus $F$ is the left dictatorship. Q.E.D.

For Nash implementation, the possibilities for individual rationality are broader: 
Theorem 10. Among the social rules $F$ satisfying the hypotheses of Theorem 5, the set of those that are individually rational, feasible and Nash implementable consists of those satisfying

$$
\sum_{i=1}^{n-1} v_{i}^{\prime}(K)+h_{n}\left(v_{1}^{\prime}(K), \ldots, v_{n-1}^{\prime}(K)\right) \geqq 0, \quad \forall K,
$$

$$
\iota_{i} \geqq-K^{*} v_{i}^{\prime}\left(K^{*}\right) \quad \text { where } \quad K^{*} \in F_{0}\left(v_{1}, \ldots, v_{n}\right),
$$

(iii) $\quad \sum_{i=1}^{n} t_{i} \leqq 0$.

Proof. Suppose that a social choice rule $F$ satisfies the hypotheses of Theorem 5 and is individually rational and feasible. From feasibility, (iii) must be satisfied. For given $v_{1}, \ldots, v_{n}$ and for $K^{*} \in F_{0}\left(v_{1}, \ldots, v_{n}\right)$, choose $\tilde{v}_{i} \in V^{*}$ with

$$
\tilde{v}_{i}\left(K^{*}\right)=K v_{i}^{\prime}\left(K^{*}\right), \quad \forall K .
$$

Because $\tilde{v}_{i}^{\prime}\left(K^{*}\right)=v_{i}^{\prime}\left(K^{*}\right)$,

$$
K^{*} \in F_{1}\left(\tilde{v}_{i}, v_{-i}\right)
$$

Thus from individual rationality,

$$
t_{i}\left(K^{*}, \tilde{v}_{i}^{\prime}\left(K^{*}\right), v_{-i}^{\prime}\left(K^{*}\right)\right) \geqq-K^{*} \tilde{v}_{i}^{\prime}\left(K^{*}\right) .
$$

Hence

$$
t_{i}\left(K^{*}, v_{i}^{\prime}\left(K^{*}\right), v_{i}^{\prime}\left(K^{*}\right)\right) \geqq-K^{*} v_{i}^{\prime}\left(K^{*}\right),
$$

establishing (ii). Summing (ii) over $i$, we obtain

$$
\sum_{i=1}^{n} t_{i} \geqq-K^{*} \sum_{i=1}^{n} v_{i}^{\prime}\left(K^{*}\right)
$$

Thus from (iii),

$$
\sum_{i=1}^{n} v_{i}^{\prime}\left(K^{*}\right) \geqq 0 .
$$

Thus, if $h$ corresponds to $F$,

$$
\sum_{i=1}^{n-1} v_{i}^{\prime}\left(K^{*}\right)+h_{n}\left(v_{1}^{\prime}\left(K^{*}\right), \ldots, v_{n-1}^{\prime}\left(K^{*}\right)\right) \geqq 0 .
$$

Varying $v_{n}$, we obtain (i). Thus (i), (ii), (iii) are necessary for individual 
rationality and feasibility. Conversely, suppose that $F$ satisfies the hypotheses of Theorem 5 and (i), (iii). From (iii), $F$ is feasible. From concavity,

$$
v_{i}(K) \geqq K v_{i}^{\prime}(K)
$$

Hence (ii) implies

$$
v_{i}(K)+t_{i} \geqq 0,
$$

establishing individual rationality. Q.E.D.

Remark. Observe that if $h=\sum_{i=1}^{n} v_{i}^{\prime}$ (so that the public decision $K^{*}$ maximizes $\left.\sum_{i=1}^{n} v_{i}\right)$

$$
-\sum_{i=1}^{n} K v_{i}^{\prime}(K)=0
$$

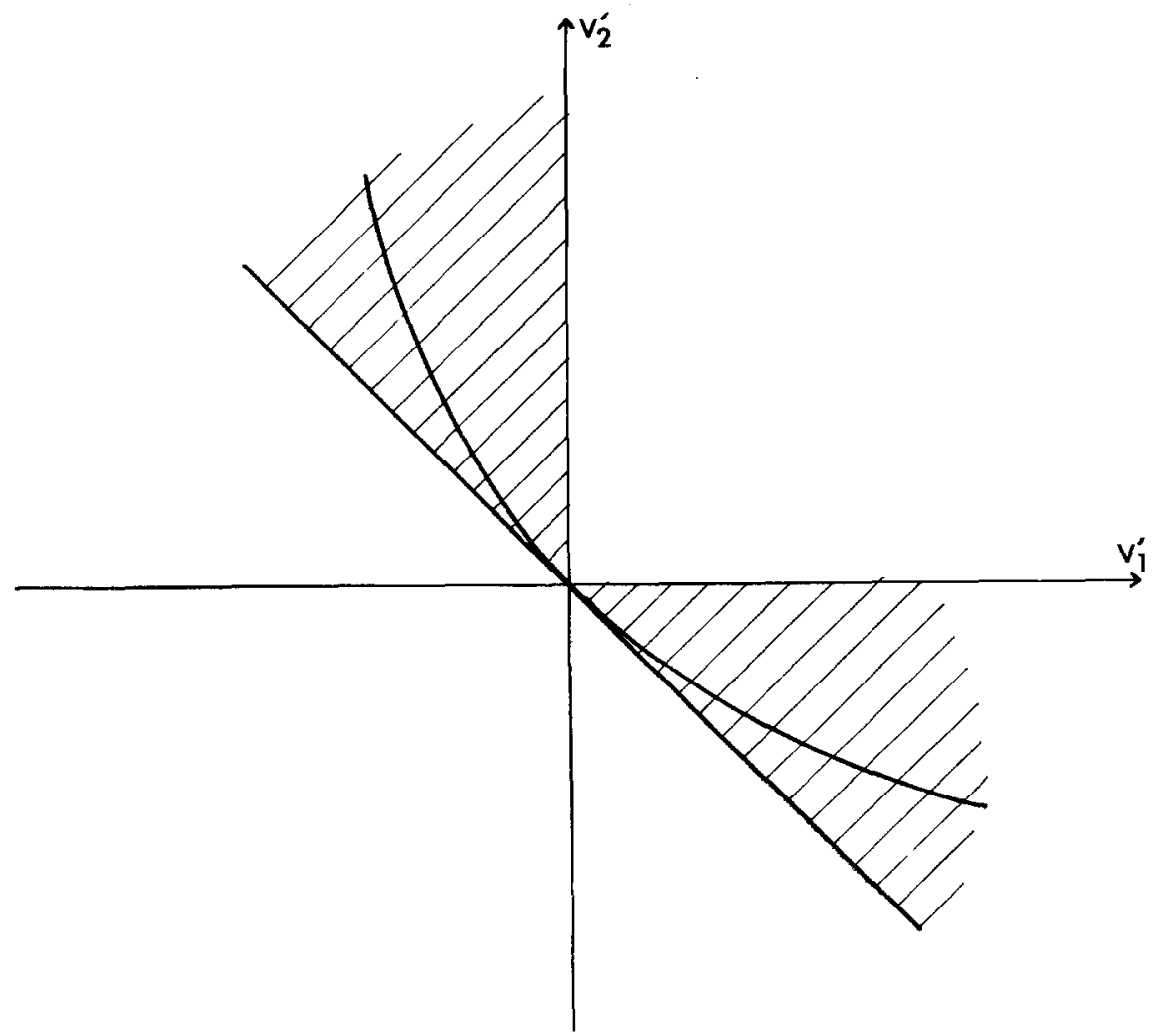

Fig. 10 
Thus if $F$ is individually rational and feasible,

$$
t_{i}=-K^{*} v_{i}^{\prime}\left(K^{*}\right)
$$

This choice of public decision and vector of transfers corresponds to the Lindahl equilibrium of the economy. That is, $F$ must be the Landahl correspondence which is Pareto optimal, yielding a result analogous to Hurwicz $(1979 b)$.

The $h(\cdot)$ functions satisfying Theorem 10 can be represented in the case of two agents as shown in fig. 10. Any scmi-strictly incrcasing $h$ function whosc zeros lie entirely in the shaded area corresponds to (in general many) feasible individually rational and Nash implementable social shoice rules.

Concluding remark. We have worked in this paper with a continuous project space $(0,1)$. However, much of the literature on incentives and public goods concerns a discrete project space - where an indivisible project is either undertaken or not - Despite their apparent dissimilarity, the two approaches are not very different in terms of the results they yield. Indeed, all our theorems have almost exact discrete analogues, as comparison with the results of Laffont and Maskin (1981, sec. 3) establishes.

\section{References}

Dasgupta, P., P. Hammond and E. Maskin, 1979, The implementation of social choice rules: Some general results on incentive compatibility, Review of Economic Studies 46, 185-216.

Gibbard, A., 1973, Manipulation of voting schemes: A general result, Econometrica 41, 587-602.

Green, J. and J.J. Laffont, 1979, Incentives in public decision making, in: Studies in public economics, Vol. 1 (North-Holland, Amsterdam).

Groves, T. and J. Ledyard, 1977, Optimal allocation of public goods: A solution to the 'free rider' problem, Econometrica 45, 783-809.

Hurwicz, L., 1972, On informationally decentralized systems, in: C.B. McGuire and R. Radner, eds., Decision and organization (North-Holland, Amsterdam).

Hurwicz, L., 1979a, Outcome functions yielding Walrasian and Lindahl allocations at Nash equilibrium points, Review of Economic Studies 46, 217-225.

Hurwicz, L., 1979b, On allocations attainable through Nash equilibria, in: J.J. Laffont, ed., Aggregation and revelation of preferences (North-Holland, Amsterdam).

Laffont, J.J. and E. Maskin, 1980, A differential approach to dominant strategy mechanisms, Econometrica 48, 1507-1520.

Laffont, J.J. and E. Maskin, 1981, The theory of incentives: An overview, in: The Aix Econometric Society world congress surveys, forthcoming.

Maskin, E., 1977, Nash equilibrium and welfare optimality, Mimeo.

Moulin, H., 1980, Strategy proofness and single peakedness, Public Choice 35, 437-455.

Roberts, K., 1979, The characterization of implementable choice rules, in J.J. Laffont, ed., Aggregation and revelation of preferences (North-Holland, Amsterdam).

Satterthwaite, M., 1975, Strategy-proofness and Arrow's conditions: Existence and correspondence theorems for voting procedures and social welfare functions, Journal of Economic Theory 10 , $187-217$

Satterthwaite, M. and H. Sonnenschein, 1981, Strategy proof allocation mechanisms at differentiable points, Review of Economic Studies 68, 580-587. 